\title{
Investigation and back-propagation modeling of base pressure at sonic and supersonic Mach numbers
}

Cite as: Phys. Fluids 32, 096109 (2020); https://doi.org/10.1063/5.0022015

Submitted: 16 July 2020 . Accepted: 02 September 2020 . Published Online: 21 September 2020

Asif Afzal (D), Sher Afghan Khan (D, Md. Tariqul Islam, R. D. Jilte (D), Ambareen Khan (D), and Manzoore Elahi M. Soudagar (D)

CrossMark

\section{Sign up for topic alerts} New articles delivered to your inbox 


\title{
Investigation and back-propagation modeling of base pressure at sonic and supersonic Mach numbers
}

\author{
Cite as: Phys. Fluids 32, 096109 (2020); doi: 10.1063/5.0022015 \\ Submitted: 16 July 2020 - Accepted: 2 September 2020 • \\ Published Online: 21 September 2020
}

\begin{abstract}
Asif Afzal, ${ }^{1, a)}$ (D) Sher Afghan Khan, ${ }^{2}$ (D) Md. Tariqul Islam, ${ }^{3}$ R. D. Jilte, ${ }^{4}$ (D) Ambareen Khan, ${ }^{5}$ and Manzoore Elahi M. Soudagar ${ }^{6}$

AFFILIATIONS

${ }^{1}$ Department of Mechanical Engineering, P. A. College of Engineering (Affiliated to Visvesvaraya Technological University), Belagavi, Mangalore 574153, India

${ }^{2}$ Department of Mechanical Engineering, Faculty of Engineering, IIUM Malaysia, 50728 Kuala Lumpur, Malaysia

${ }^{3}$ School of Chemical Engineering, The University of Queensland, Brisbane, QLD 4072, Australia

${ }^{4}$ Department of Mechanical Engineering, Lovely Professional University, Punjab 144411, India

${ }^{5}$ School of Aerospace Engineering, Universiti Sains Malaysia, 14300 Nibong Tebal, Penang, Malaysia

${ }^{6}$ Department of Mechanical Engineering, Faculty of Engineering, University of Malaya, Kuala Lumpur 50603, Malaysia
\end{abstract}

a) Author to whom correspondence should be addressed: asif.afzal86@gmail.com

\begin{abstract}
The experimental analysis of base pressure in a high-speed compressible flow is carried out. The flow is made to expand abruptly from the nozzle into an enlarged duct at fifteen sonic and supersonic Mach numbers. The analysis is made for variation in the nozzle pressure ratio (NPR), length to diameter ratio, and area ratio. The effect of active micro-jets on the base and wall pressure is assessed. The data visualization of the huge experimental data generated is performed using heat maps. For the first time, six back-propagation neural network models (BPMs) are developed based on input and output possibilities to predict the pressure in high-speed flows. The experimental analysis revealed that depending upon the type of expansion, the base pressure changes. A jet of air blown at the base using micro-jets is found to be effective in increasing the base pressure during the under-expansion regime, while the wall pressure remains unaffected. The data visualization provided an insight into the highest impact on the base pressure by the NPR. The six BPMs with two hidden layers having four neurons per layer are found to be most suitable for the regression analysis. BPM 5 and BPM 6 accurately predict the highly non-linear data of the base and wall pressure.
\end{abstract}

Published under license by AIP Publishing. https://doi.org/10.1063/5.0022015

\section{NOMENCLATURE}

ANN artificial neural network

BPM back-propagation model

CFD computational fluid dynamics

$\mathrm{L} / \mathrm{D}$ length to dimeter ratio

$\mathrm{M} \quad$ Mach number

NN neural network

NPR nozzle pressure ratio

$\mathrm{P} \quad$ pressure

WC with control
WoC without control

$\mathrm{X} / \mathrm{L}$ duct location to length ratio

\section{Greek symbols}

$\phi_{r} \quad$ length to dimeter ratio

$\Lambda_{r} \quad$ area ratio

$\xi_{r} \quad$ nozzle pressure ratio

$\Psi_{p} \quad$ wall pressure

$\Omega_{b} \quad$ base pressure

$\gamma \quad$ ratio of specific heat 


\section{INTRODUCTION}

A sudden increase in area results in flow separation and reattachment. High-speed detached flows are not entirely realized despite significant work that has been done. To date, we are unable to predict these flows. These flows are difficult to predict precisely due to intricate fluid dynamic phenomena. This includes the presence of shock waves, expansion waves, and large flow property gradients. $^{1-8}$ The shear layer that is exiting from the nozzle results in recirculating flow regions with sub-atmospheric pressure at the base pressure at large Reynolds numbers, turbulent, and highly compressible shear flows. Insight into the fluid dynamic mechanisms in the near-field region at the back of the axis-symmetric bodies is critically important for foretelling high-speed rocket and performance of the aerospace vehicles. Exclusively, the formation of a low-pressure re-circulation zone acting on the rear-looking base of the cylinder-shaped blunt bodies at transonic and supersonic Mach numbers can contribute considerable amounts to the base drag values. It is found that the base drag values at transonic Mach numbers are around $60 \%$ of the total drag of the aerospace vehicles. $^{9-12}$

Korst $^{13}$ investigated the base pressure issue in the transonic and supersonic flow regime, where the external flow at the base was sonic and likewise supersonic after the wake. The author developed a physical model by considering the interaction between the adjacent jet and the dissipative shear flow in the wake. Similarly, the sudden expansion of air has been investigated in a cylindrical duct for the case of noise production and base pressure, ${ }^{14}$ which was minimum because of the attached flow that depended on the ratio of duct and nozzle area. Khan and Rathakrishnan ${ }^{2}$ have examined the boundary layer control by decreasing the drag in full submerged bodies. The authors utilized the suction technique on the boundary layer; therefore, the pressure improved from the axisymmetric bodies resulting in separation control. The sudden expansion of fluid flow in a pipe was investigated in Ref. 1. It was reported that the normalized base pressure depends on the ratio of pressure (overall), area, length to diameter $\left(\phi_{r}\right)$, and expansion of the duct. It was found that the ratio of area and overall pressure and an optimal ratio of $\phi_{r}$ showed maximum pressure at the exit of the nozzle, while the minimum base pressure was near the sudden expansion plane.

Similarly, the distribution of pressure near the wall for a low supersonic Mach number $(M=1.3)$ was investigated. ${ }^{15}$ The expansion and compression waves decreased significantly at $\phi_{r}=3$ due to the back-pressure difference. The interesting results were that the pressure near the wall was negligible under expanded flows for both with and without control (WoC) cases, and the effect remained constant. ${ }^{16}$ Also, experimental investigations were performed for underexpanded and correctly expanded cases in the duct at $M=1.25$ and a diameter ratio of nozzle of 1.6. ${ }^{17}$ It was found that the flow became oscillatory for a distinct arrangement of the nozzle pressure ratio $(N P R)$ and $\phi_{r}$. The oscillatory nature was found for both with control (WC) and without control cases. Several research works have also been carried out by using an active/passive control system to control separation. ${ }^{18-2}$

On these types of problems, soft computing is a new technique that parallels the amazing capability of the human mind to aim and study in an environment of uncertainty and imprecision. ${ }^{2}$
It comprises several computing models, which include the fuzzy set theory, neural networks, an optimization technique, approximate reasoning, clustering, classification, regression, and so on. ${ }^{25,26}$ A fuzzy logic approach is found in the literature for the investigation of pressure loss in a sudden expansion duct with a de Laval nozzle. ${ }^{27}$ The authors focused on minimum pressure loss that takes place when $\phi_{r}$ is 1 , which was obtained by using the fuzzy logic formulation. The values of $M$ were $1.58,1.74,2.06$, and 2.23, while the values of area ratio $\left(\Lambda_{r}\right)$ and pressure ratio were $10.00,6.00,2.89$, and 2.65, respectively. The authors stated that the fuzzy logic approach could assist in quantifying the internal fluid flow through a nozzle with a sudden expansion in the duct. Similarly, Pandey and $\mathrm{Kumar}^{28}$ investigated the flow at $M=2.4$, which is higher than the previous study. However, the values of pressure ratio were $2.10,2.65$, and 3.48 for $\Lambda_{r}=2.89$ and $\phi_{r}=6-1.24$. The authors found that the results using the fuzzy logic theory are suitable for smooth flow development compared to all other parameters at $\phi_{r}=4$. It evaluated the optimum value of $\phi_{r}$ from a sudden expansion circular duct for $M=1.58$, $1.74,2.06$, and 2.23 , the primary pressure ratio of $2.10-3.48$, and $\Lambda_{r}$ of $2.89,6.00$, and 10.00 . Based on the evaluation, the optimum value of $\phi_{r}=6$ was found to be desirable for all other parameters such as the wall static pressure and loss pressure, including the base pressure. $^{29}$

The fuzzy logic method applied in a circular duct without cavities for the case of $M=1.74$ and $2.23^{30}$ and cavities for the instance of $M=1.58-2.06^{31}$ to examine the wall static pressure variation on the smooth fluid flow condition. The authors selected three $\Lambda_{r}(2.89$, 6.00 , and 10.00), $\phi_{r}=1,2,4$, and 6 , a pressure ratio of 2.65 , and the cavity aspect ratio of 1 and 2 . The de Laval nozzle was used for the case of without cavities, while the conical nozzle was used for the cavities cases. In both cases, the fuzzy logic approach was acceptable for smooth flow based on wall static pressure variations at $\phi_{r}=1$. The authors have successfully utilized an artificial neural network (ANN) to estimate the floor pressure in a convergent nozzle with shallow cavity internal flows at different subsonic Mach numbers.

Similarly, ANN has also been used in civil engineering applications to detect the bursts and other abnormal flows at a district meter area level. ${ }^{32}$ ANN and a mixture density network were trained through the updated database of the future flow profile. On the other hand, flow characterization over a transport airplane configuration was learned and trained with different sizes of the network to predict aerodynamic coefficients by using ANN. ${ }^{33}$ The authors carried out experimental work and compared it with ANN to investigate its potential for the conceptual design process. In order to obtain the lift coefficient, the lower and upper boundaries of airplane configurations were selected at the subsonic $M$ of 0.2 and 0.8 , respectively. At the same time, the other parameters of each configuration for the upper and lower boundaries were chosen by using ANN input variables. The ANN method was used to predict the lift coefficients for different angles of attack at a specified Reynolds number and flow separation in a NACA0012 airfoil. ${ }^{34}$ Comparing with the computational fluid dynamics (CFD) approach, it was found that the ANN method can predict the lift coefficient for a preliminary design at a considerably low cost of computational study.

Several studies were carried out to optimize the base pressure in a nozzle flow through the sudden expansion duct with and without control. Of those studies, the design of experiments (DOE) approach 
allows optimizing the effect of the parameters on control of the fluid flow process. For example, the DOE method was used to analyze the suddenly expanded flow with and without micro-jets active control for $M=2$ and $3, N P R=5$ and $9, \phi_{r}=3.25$ and 6.25 , and $\phi_{r}=4$ and 8 as the minimum and maximum values, respectively. ${ }^{35}$ The authors used a full factorial design of the L16 orthogonal array (OA) to develop the linear model. It was found that the linear regression models for base pressure with and without control are statistically suitable and accomplished in making precise estimations. The same authors ${ }^{36}$ presented the study for three levels from each parameter, which is similar to the previous one. The low-medium-high levels and the medium range were set for $M=2.5, N P R=7, \Lambda_{r}=4.75$, and $\phi_{r}=6$. The L27 orthogonal array was implemented to optimize the results using response surface methodology. A non-linear model based on the $\mathrm{C}-\mathrm{C}$ (central composite) and B-B (Box-Behnken) design was proposed to ease the input and response correlation. Using the ANOVA (analysis of variance) table, the significance of square, main, and interaction terms of the proposed models was analyzed.

The base pressure analysis in the field of suddenly expanded compressible flow into the duct at sonic and supersonic Mach numbers is rarely reported. Additionally, the use of passive or control mechanisms is a potential area to regulate the base pressure in the duct base. Regression using ANN in this field can be said as new, which must be investigated. This article examines the potential of dynamic control by applying micro-jets to increase the base pressure at sonic and supersonic Mach numbers. A comparison between the controlled and normal flow affected by operating parameters and geometry of the duct is studied. A completely new contribution in this high-speed flow study is the development of different backpropagation (BP) ANN models and correlations to predict the pressure at the base and duct wall. The data analysis is also performed to understand the level of effect of parameters on the response variable.

\section{EXPERIMENTAL SETUP}

The sudden expansion flow characteristics such as the recirculation zone, reattachment point, and expansion waves are investigated experimentally, and it is presented in Fig. 1. The schematic view of the experimental setup of sudden expansion flow investigation in a duct is shown in Fig. 2. The settling chamber and nozzle with a duct arrangement is shown in Fig. 2(a). The upstream nozzle arrangements connecting pressurized tanks to the nozzle through the steeling chamber are shown in Fig. 2(b). The complete setup with pressure transducer arrangements and a data acquisition system (DAQ) is shown in Fig. 2(c). It consists of a nozzle that is an arrangement of four micro-jets at the base, which has eight holes along with the circular position, as shown in the right-side view of Fig. 2. The holes of the micro-jet are denoted with " $c$ " and placed for blowing of air, while the holes " $\mathrm{m}$ " are meant for measuring the base pressure $\left(\Omega_{b}\right)$. Here, the hole " $c$ " is used to control the base pressure, and it is connected with the blowing chamber by using a tube. The pressure of the blowing chamber is the same as in the settling chamber. Pressure taps are used to measure the wall pressure and the flow field nature in the duct. The distance between " $\mathrm{m}$ " and " $\mathrm{c}$ " holes is $8 \mathrm{~mm}$, while it is $10 \mathrm{~mm}$ in between " $c$ " and " $\mathrm{m}$ " holes. The ranges of the length to diameter ratio $\left(\phi_{r}=L / D\right)$ are from 10 to 1 , and

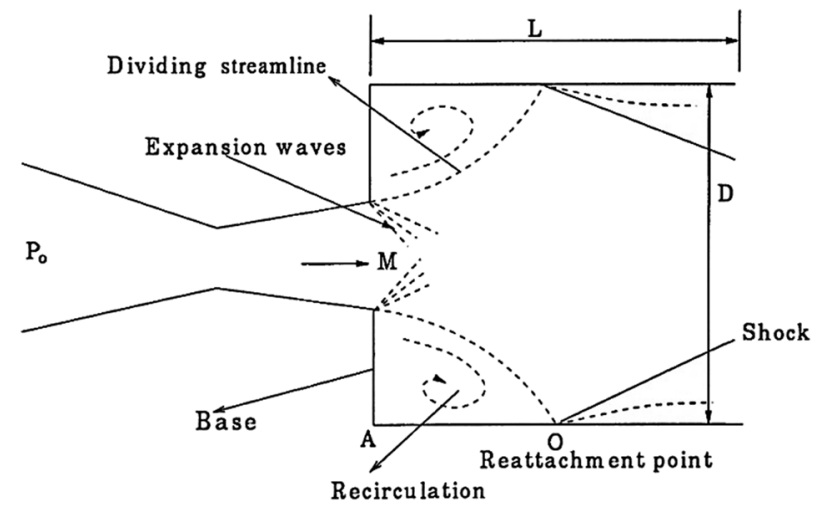

FIG. 1. Different zones of the sudden expansion flow field characteristics.

readings for each $\phi_{r}$ are measured. The experiment is performed for 15 Mach numbers, i.e., sonic Mach 1 and supersonic Mach 1.25, 1.3, $1.48,1.6,1.7,1.8,1.87,2,2.2,2.3,2.5,2.58,2.7$, and 3. Typically, the range of $\phi_{r}=3-5$ has been considered for the case of without control; however, $\phi_{r}$ can vary from 10 to 1 for the case of control. The nozzle pressure ratio $\left(\xi_{r}\right)$ is varied from 1 to 10 in a step of 1 each for every $\phi_{r}$, and readings are taken for every single time. The base pressure variation is recorded by using a pressure transducer (PSI System 2000), and the range of pressure is up to 300 psi (15 bars), and it has 16 channels. The sampling rate of the pressure transducer is $250 \mathrm{samples} / \mathrm{s}$, and the wall pressure is recorded by using a mercury manometer. The pressure transducer has 16 channels, out of which channels 1 and 2 is used to measure the stagnation pressure in the main settling chamber, and the channel 2 measures the stagnation pressure in the control chamber. The remaining 14 ports were used to measure the wall pressure of the enlarged duct. Hence, 14 wall pressure taps were connected to the pressure transducer, and the rest of the wall pressure taps were connected with the multi-tube manometer. Therefore, all the fluctuations that take place within the reattachment length are measured by the pressure transducer. The photographic view of the experimental test rig, pressure tanks, and wall pressure manometers is shown in Figs. 2(d)-2(f).

\section{BACK-PROPAGATION (BP) ANN}

The back-propagation (BP) algorithm is the most fundamental building block in a neural network (NN). It is utilized to efficiently train a NN through a technique known as chain rule. The backward pass in BP algorithm improves its performance by adjusting the weight and biases variables. In BP, the input data are provided into the NN with the aim of classification or prediction of the data. In this case, the information is split into binary signals for allowing a single neuron. A set of known values is utilized for training the NN as the training data. The NN output can be a real value between zero and one, discrete value, or a Boolean. Each neuron receives the input and passes to the activation functions, which are the tanh, ReLU, and sigmoid functions. The active functions stabilize the predicted value to a specified range in order to safeguard the model and have stable convergence. 


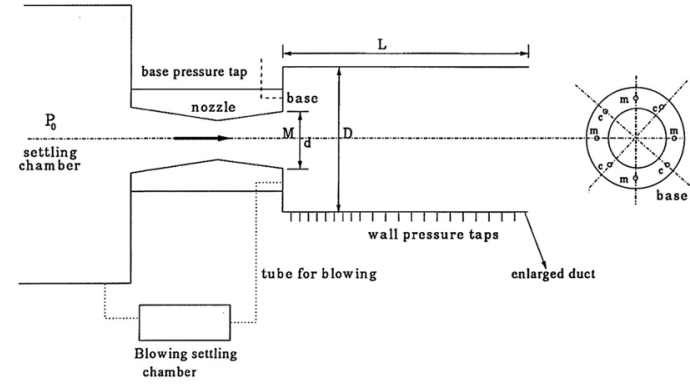

(a)

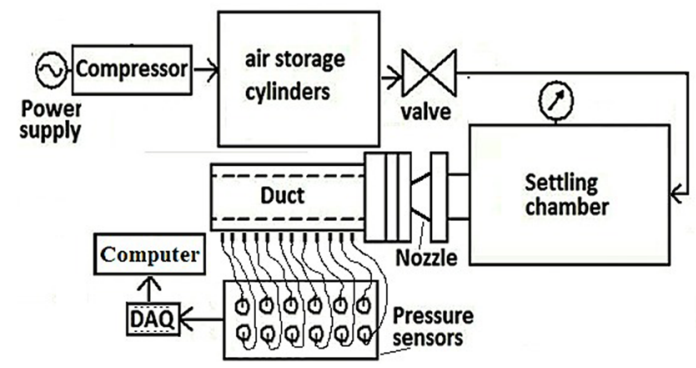

(c)

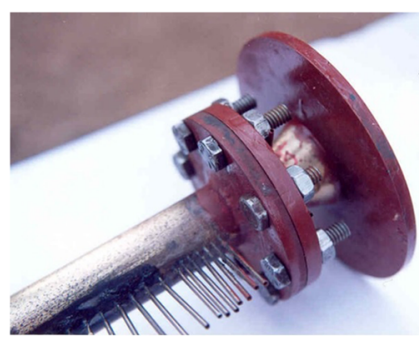

(e)

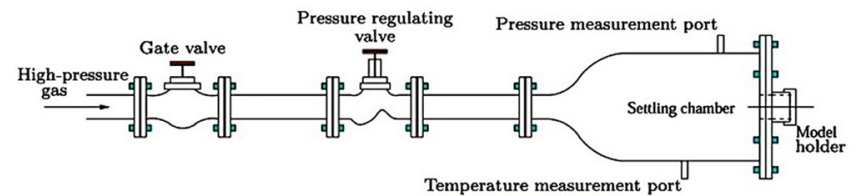

(b)

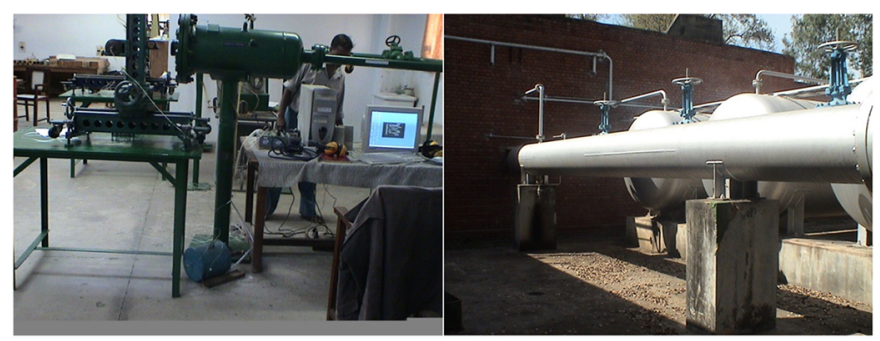

(d)

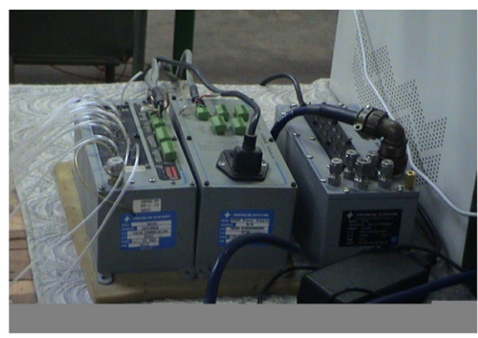

(f)

FIG. 2. Experimental setup, nozzle, and duct arrangement for the sudden expansion flow analysis (a) Nozzle and duct arrangements with micro-jets. (b) Flow of compressed gas through different values to the nozzle holder. (c) Complete setup of compressible flow flowing through tanks to the duct. (d) Setup and pressure tanks. (e) Duct wall manometers. (f) Pressure transducer.

Typically, a numeric weight is introduced to each neuron for the determination of each neuron's output. In the BP training model, the aim is to determine the weights that can create the maximum precise output and are set at the beginning before the model is trained. First, the NN initializes the weights arbitrarily and then proceeds in optimizing from there. For example, Xavier optimization ensures proper initialization of weights to generate sufficient signals that pass from all layers of the network. In the BP model, the forward pass attempts are made by taking the inputs which pass from the network to allow reaction of each neuron with the fraction input, ultimately producing an output. In the BP model, gradient descent is a mathematical formulation that modifies the function variables from a higher to lower value by looking at the derivatives of the function about each of its variables and that sighted which step, through which variable, is the following appropriate phase to minimalize the function. Gradient descent is applied to the error function, which helps us to find the lowest weights and make the model more accurate. The RMSE (root mean squared error) is estimated and can be written as follows to train the model:

$$
R M S E=\sum_{i=1}^{N} \sqrt{\frac{\left(x_{i}-\hat{x}_{i}\right)^{2}}{N}},
$$

where $x$ and $\hat{x}$ are the observed and predicted quantities, respectively, $N$ is the number of data for RMSE calculation, and $i$ is the data index.

The BP model (BPM) developed in this regression study is of different types. A general representation of the BPM with inputs is Mach, $\xi_{r}$ (NPR), $\Lambda_{r}$ (area ratio), $\phi_{r}(L / D)$, control (0-without or 1with), and non-dimensional length $X / L$. The outputs are the base pressure $\Omega_{p}$ and wall pressure $\Psi_{p}$, which are shown in Fig. 3. The number of layers and neurons per layer are tried to get minimum RMSE. The neurons per layer are tested from 3 to 9 , and the number of hidden layers is tested from 1 to 3 . The wall pressure is measured 


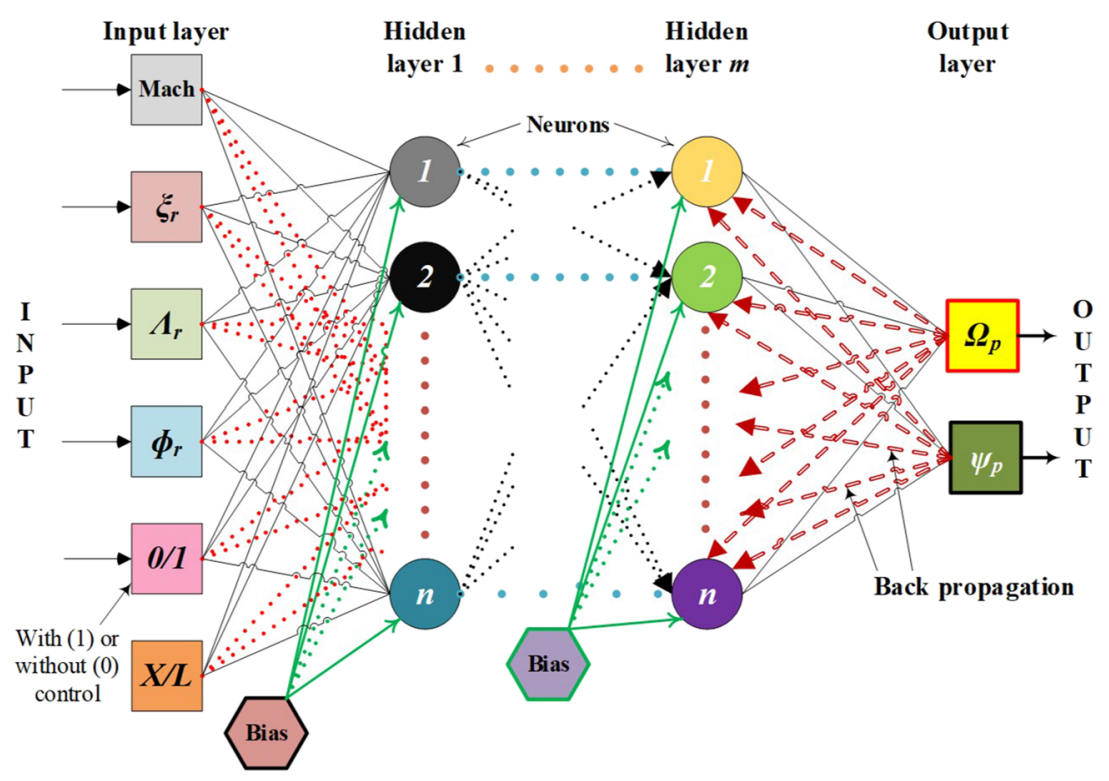

FIG. 3. A general schematic representation of the back-propagation model (BPM) used for modeling pressure (base and wall) in different conditions.
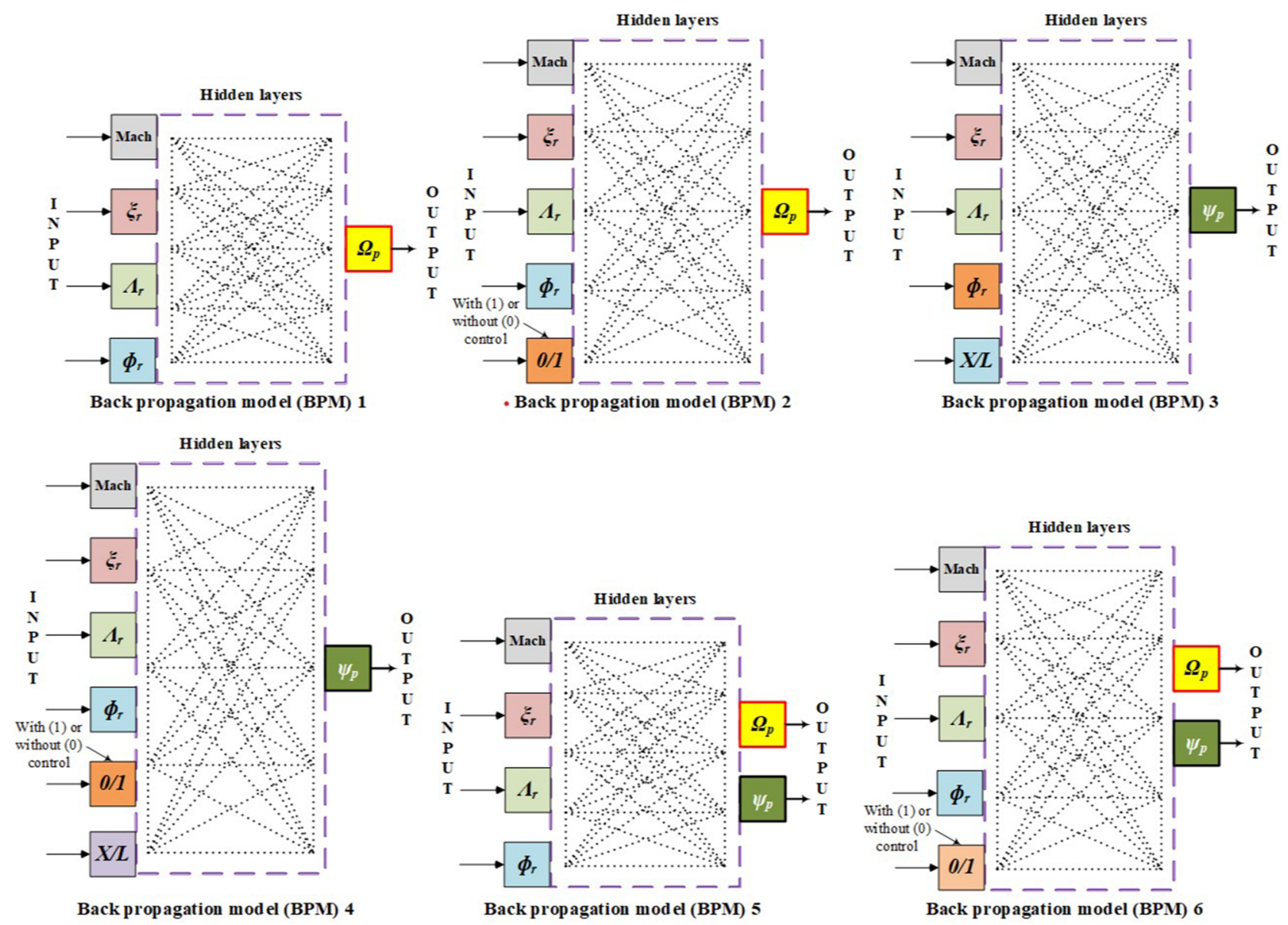

FIG. 4. Six BPMs developed in this study for the regression of base and wall pressure with (WC) and without control (WoC). 
along the complete length of the duct $(X / L)$, while the base pressure is only read at the base. Therefore, the BPM regression is divided into primarily three types of regression, such as for (1) only base pressure, (2) only wall pressure, and (3) both the pressures. In the case of regression type 3 , the wall pressure only at one location, i.e., next to the base (common to all $\phi_{r}$ ), is selected so that it can be predicted combined with the base pressure as it is also only one reading. The BPM developed for these regression types is shown in Fig. 4, where a total of six models are proposed. As in this study, the flow is regulated with control (1), and without control (0), and few BPMs are tested for both cases like BPM 1, BPM 3, and BPM 5. Remaining models are trained using combined with and without control pressure data, which are BPM 2, BPM 4, and BPM 6. In these models, controls ( 1 and 0$)$ are input to the network. As mentioned earlier, the BP models are tested, and the optimized network for each BPM is used to predict or do testing of data, which is $10 \%$ of total data. The base pressure data are nearly 6000 points combining control and no control. The wall pressure data combined are almost $0.15 \times 10^{6}$; therefore, this BP modeling is most suitable to carry out regression for these vast data. Apart from this, two correlations for highly nonlinear base pressure are also developed, and a comparison is made with the BPM.

\section{RESULTS AND DISCUSSION}

\section{A. Pressure analysis}

The present study aims to investigate the impact of the dynamic control on the base pressure $\left(\Omega_{p}\right)$. The study also seeks to examine the influence of the control mechanism on the flow development in the enlarged duct. The back-pressure normalized the experimental data obtained like the base pressure and the static wall pressure $\left(\Psi_{p}\right)$ in the conduit. The base pressure is minimal (i.e., less than the ambient pressure) whenever there is an abrupt change in the area of the duct. The sudden increase in the area results in flow separation and reattachment with the duct wall, and later again, there will be a boundary layer growth. The Mach numbers considered during tests were in the range of 1-3. Experiments were conducted for $\xi_{r}$ from 3 to 11. From the range of the inertia parameters, it is evident that up to Mach 1.8, the flow from the nozzles is perfectly and under-expanded. For Mach 2, the exiting flow from the nozzle is correctly, under-, and imperfectly expanded. For correct expansion, $\xi_{r}$ for a particular Mach number is calculated by the following equation:

$$
\xi_{r}=\frac{P_{o}}{P_{a}}=\left(1+\left(\frac{\gamma-1}{2}\right) M^{2}\right)^{\frac{\gamma}{\gamma-1}}
$$

The flow behavior in these expansion types is schematically shown in Fig. 5. For Mach numbers 2.5 and 3, the flow is over-expanded. The base pressure results for all the Mach numbers are shown in Fig. 6, but the $\xi_{r}$ range selected was 3, 5, and 7. $\Lambda_{r}$ and $\phi_{r}$ are 2.56 and 10. Since for Mach numbers, $M=2.5$ and 3.0, the tests were conducted for the imperfectly expanded case. Hence, we intend to look for the effectiveness of dynamic control (WC) under the influence of an adverse pressure gradient. The intention of selecting this $\xi_{r}$ was to assess the performance of the control mechanism and to simulate the trajectory of launch vehicles. At the launch point, over-expanded jets are preferred. After the launch with the increase in the altitude, the over-expanded nozzle will become correctly expanded. In Fig. 6, it is seen that at $\xi_{r}$ (a)

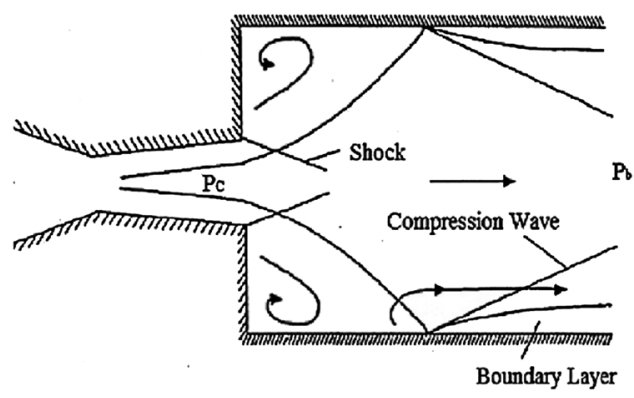

(b)

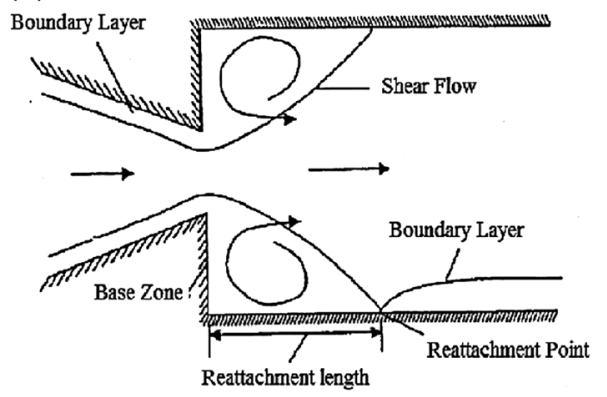

(c)

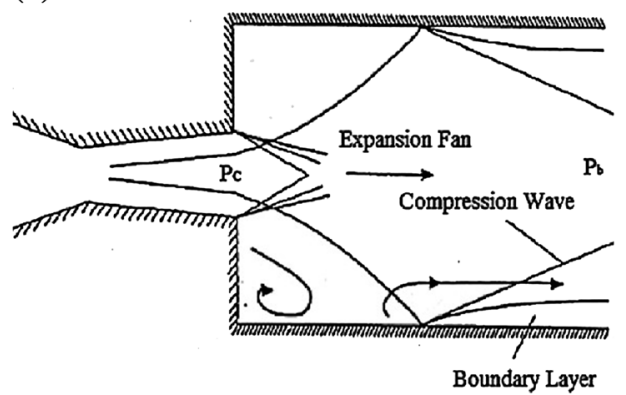

FIG. 5. Structure of (a) over-expanded (b) correctly expanded, and (c) underexpanded flows at different levels of jet expansion in the duct. 


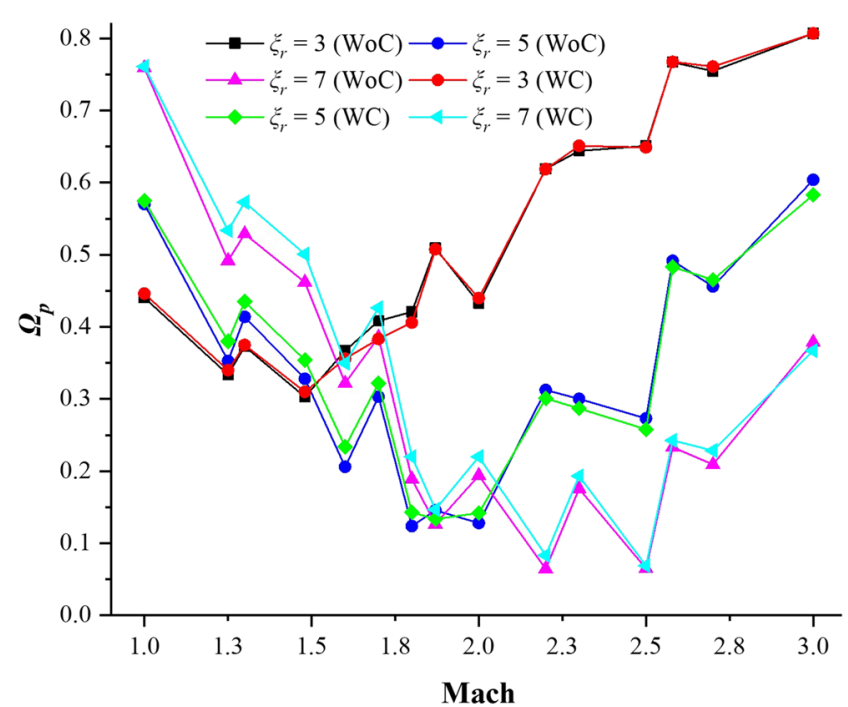

FIG. 6. Base pressure $\left(\Omega_{p}\right)$ vs Mach number $(M)$ without control (WoC) and with control (WC) systems for different $\xi_{r}=3,5$, and 7 at fixed $\Lambda_{r}=2.56$ and $\phi_{r}=10$ in sonic and supersonic flow conditions.

$=3$ and $M=1$, the flow is under-expanded, and hence, it undergoes expansion, and there is a progressive decrease in the base pressure in the absence of control. Under these circumstances, when the control mechanism (WC) is activated, there is an increase in the base pressure in the presence of a favorable pressure gradient. The trend of decreasing base pressure continues until $M=1.25$; however, with a further increase in the inertia level, the trend is reversed up to $M$ $=1.3$. Later, in the Mach number range 1.3-1.48, the decrease in the base pressure revisits. For the case of $M$ higher than 1.48, there is a sudden increase in the base pressure. It is evident at $\xi_{r}=3$ that the flow from the nozzles at $M$ beyond 1.48 will over-expand, and this level of over-expansion will increase with the Mach number, and this phenomenon is clearly seen since these jets are confined in a duct of diameter $16 \mathrm{~mm}$. In view of the small diameter, the reattachment length will be short. The shear layer exiting from the nozzle will be associated with the oblique shock wave, expansion wave, and Mach wave (Fig. 5) depending on $\xi_{r}$ and $M$. The entire flow field is dominated by the presence of waves, and hence, the flow will remain oscillatory in nature.

Similar trends are seen at $\xi_{r}=5$ and 7 . The increase in the $\xi_{r}$ values from 3 to 5 and from 5 to 7 will make the flow up to $\mathrm{M}=1.6$ as under-expanded. The jets are highly under-expanded at lower $\xi_{r}$. It is seen that whenever the nozzles are under the influence of a favorable pressure gradient (i.e., under-expanded) and when the dynamic control (WC) is activated, results in an increase of the base pressure and the revised trends are seen when the jets are overexpanded. Similar patterns are observed for $\xi_{r}=7$. Hence, based on the above results, we can say that we cannot generalize the trends due to the excessive interaction and domination of the waves inside the duct.

Figure 7 shows the base pressure variation with Mach numbers for three $\Lambda_{r}$, namely, 2.56, 4.84, and 6.25 for fixed $\xi_{r}=5$ and $\phi_{r}=10$, and the base pressure results are compared with control (WC) and

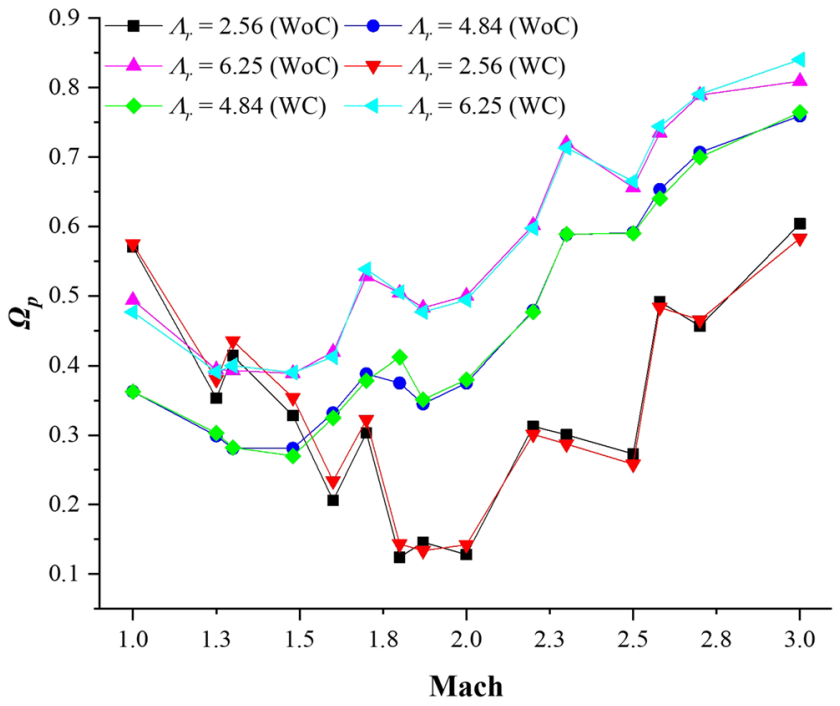

FIG. 7. Base pressure $\left(\Omega_{p}\right)$ vs Mach number $(M)$ without control (WoC) and with control (WC) systems for different $\Lambda_{r}=2.56,4.84$, and 6.25 at fixed $\xi_{r}=5$ and $\phi_{r}=10$ in sonic and supersonic flow conditions.

without control (WoC). Here, the effect of $\Lambda_{r}$ (i.e., the relief available) on the flow is dominating the base pressure values. In Fig. 7, it is seen that in the case of the lowest $\Lambda_{r}$, the base pressure is increasing with $M$ from 2 and above. However, the same trend is seen from $M$ $=1.5$ and above. However, the pattern of increasing the base pressure starts from $M=1.3$. When the control was activated at lower $M$ and $\Lambda_{r}=2.56$, the micro-jets are able to reduce the base suction significantly as long as the flow from the nozzles is under-expanded for $M>1.6$, and the control results in a reduction of the base pressure. The reasons for this trend may be due to the variable level of expansion. The jets are under-expanded until $M=1.6$, and for remaining Mach numbers, the jets are over-expanded, and the level of over-expansion increases with the Mach number significantly. The location of the control jets is in the middle of the base region as the $p c d$ (pitch circle diameter) of the control jets was fixed at $13 \mathrm{~mm}$. Hence, the control effectiveness will have variable trends at other $\Lambda_{r}$, namely, 4.84 and 6.25 due to the proximity of the control jets very close to the base corner and away from the main jet. For $\Lambda_{r}=6.25$, it will further move away from the main jet while discussing the base pressure results that the reattachment length will increase with an increase in $\Lambda_{r}$. Hence, for the same $M, \phi_{r}$, and $\Lambda_{r}$, the base pressure will increase with the rise in $\Lambda_{r}$. The reason for a given/fixed value of $\xi_{r}, \phi_{r}$, and $M$ is that the reattachment will continue to grow. In this situation, the vortex strength, which is fixed, can create powerful suction in the base region, and at higher $\Lambda_{r}$, the effectiveness of the base vortex gets reduced. Hence, based on the above discussions, these trends can be understood. The control effectiveness is marginal at higher $\Lambda_{r}$.

Figure 8 shows the base pressure results at sonic and supersonic Mach numbers for $\phi_{r}=2,6,10 \mathrm{WC}$ and WoC for $\xi_{r}=5$ and $\Lambda_{r}$ $=2.56$. The results show that $\phi_{r}=2$ is insufficient for the flow to remain attached for $M>1.6$. Since the base pressure results considered are for fixed $\xi_{r}=5$. The flow exiting from the nozzle is 


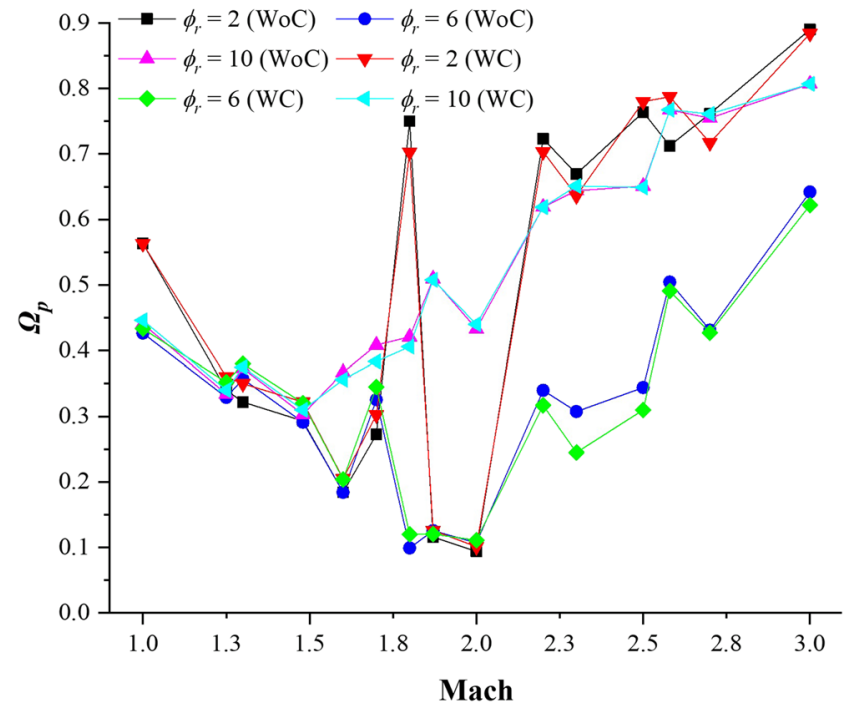

FIG. 8. Base pressure $\left(\Omega_{p}\right)$ vs Mach number $(M)$ without control (WoC) and with control (WC) systems for different $\phi_{r}=2,6$, and 10 at fixed $\xi_{r}=5$ and $\Lambda_{r}=2.56$ in sonic and supersonic flow conditions.

over-expanded; hence, the base pressure assumes higher values not only due to the flow being over-expanded but also due to the insufficient length of the duct, which exposes the flow to the ambient pressure making the base pressure almost equal to the ambient atmospheric pressure. This phenomenon is visible when we see the results at $\phi_{r}=2$ for $M>1.6$, and this increase in the base increases with the Mach number. When $\phi_{r}=6$, even though the flow is attached for all the Mach numbers, the fluctuations and increase in the base pressure are attributed to the level of expansion, the interaction of the shock waves in the duct, and the influence of the Mach number. It is well known that the base pressure will increase with the increase in the inertia levels. The base pressure trends are similar for all $\phi_{r}$ up to $M=1.6$ since the flow is attached with the duct wall, and minor variation is expected due to the variation in the duct length and the effect of the back-pressure.

Figure 9 shows the base pressure for the jets at a different level of expansion (i.e., over-expansion, correct expansion, and underexpansion) at selected $M=1.87$ and 2 for the cases of WC and WoC at $\phi_{r}=10$ for $\Lambda_{r}=2.56$. However, $\xi_{r}$ for $M=1.87$ and 2 are 3.56 and 2.13 , respectively; these $\xi_{r}$ correspond for a level of over-expansion of 0.56 at $M=1.87,0.277$, and 2.0 , while $\xi_{r}$ for correct expansion are 6.97 and 7.83. Similarly, $\xi_{r}$ for a fixed level of under-expansion of 1.5 , they are 9.6 and 11.85 at $M=1.87$ and 2 . These $\xi_{r}$ and the level of expansion were selected to assess the effectiveness of the control mechanism in the form of micro-jets. As expected, when jets are over-expanded, and the Mach number is also increasing, the results show that with the increase in the level of over-expansion and Mach number, a high value of the base pressure is achieved. Under these circumstances, when the dynamic control is employed, the control results in a decrease in the base pressure. Hence, these results reiterate that when the controls are activated in the presence of an adverse pressure gradient, it results in a reduction of the base pressure.

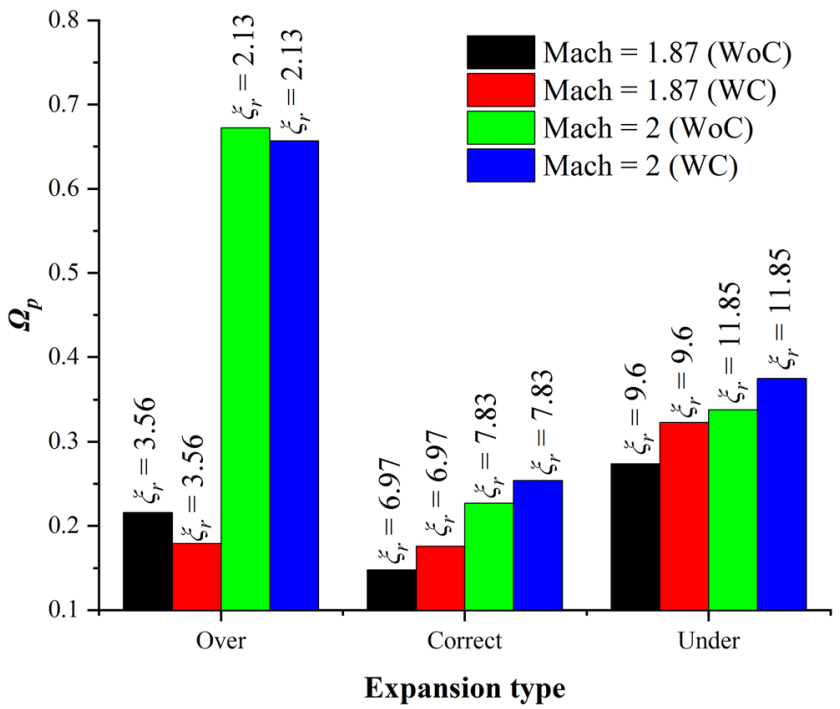

FIG. 9. Base pressure $\left(\Omega_{p}\right)$ for the jets with over-expansion, correct expansion, and under-expansion at Mach numbers $M=1.87$ and 2 for the cases of WC and WoC, while $\phi_{r}=10$ and $\Lambda_{r}=2.56$ are kept constant. $\xi_{r}$ for these expansion types are mentioned as labels over the bars.

When the jet is correctly expanded, the shear layer exiting from the nozzle will be associated with Mach waves. These Mach waves are weak waves through which the flow is isentropic. It is also seen that when the control is activated, they influence the base pressure positively, and the control results in an enhancement in the base pressure. The magnitude of the increase in the base pressure is nearly the same, unlike the over-expanded jet, where the level of overexpansion is different. When we look at the base pressure results, it is found that when the jets are under-expansion, the control increases the base pressure. Furthermore, an increase in NPR influences the level of expansion and the magnitude of the base pressure at various Mach numbers.

Figure 10 shows the results of the base pressure for correctly expanded jets at supersonic Mach numbers. The base pressure results are compared with and without control for $\Lambda_{r}=2.56$ and $\phi_{r}=10$. From the results, it is seen that for low $M=1.25$ and 1.3, the base pressure values for without control are marginal as compared to $M>1.3$, while for the lower Mach numbers, the control effectiveness is marginal. It is also seen that with the increase in the Mach number, there is a continuous decrease in the base pressure values for without control cases for Mach numbers up to 1.87. When the control by the micro-jets is employed, there is a substantial increase in the base pressure values. At $M=2.0$ and 2.2, there is an increase and decrease in the base pressure value in the absence of the control. However, the control effectiveness increases from all $M>1.5$. This trend may be due to the combined effect of the area ratio, the Mach number, and interactions of the waves at the nozzle exit as well as in the enlarged duct. The flow field is very complicated due to the sudden expansion of flow, which can form oblique shock if the flow is over-expanded and Mach waves if the flow is under-expanded, in front of the nozzle. Since the tubes were made of brass, therefore, it was not possible to visualize the flow. If the duct is of rectangular cross section and 


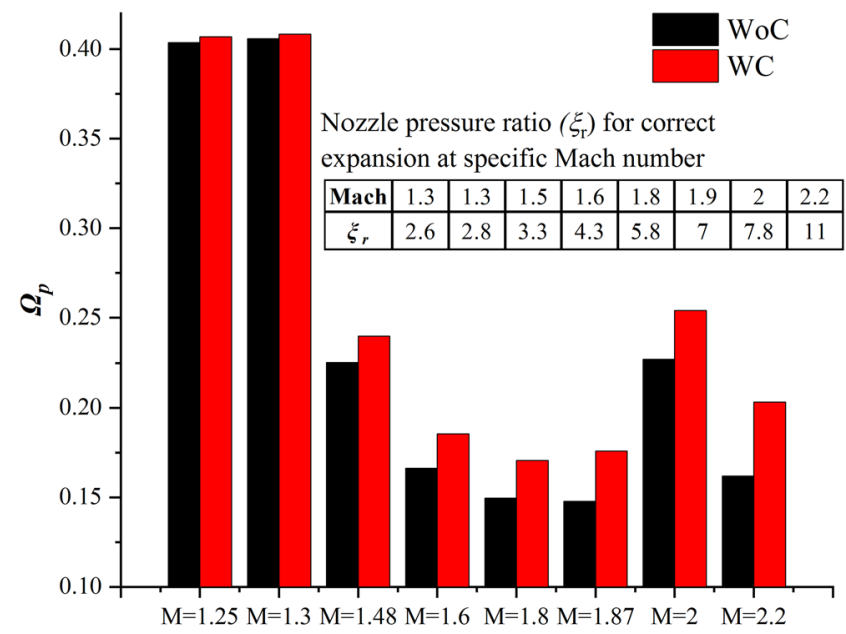

Mach

FIG. 10. Base pressure $\left(\Omega_{p}\right)$ for correctly expanded nozzle flows at supersonic Mach numbers with control (WC) and without control (WoC). The nozzle pressure ratios $\xi_{r}$ for correct expansion are mentioned in the table in the inset of this figure, while $\Lambda_{r}=2.56$ and $\phi_{r}=10$ are fixed.

the front wall is transparent, then through the shadowgraph, we can capture the shock wave pattern. In the conduit, the flow will get reattached with the wall before passing through the waves. This may be the reason for this trend. Usually, it was presumed that when the jets are correctly expanded, the flow is smooth and the flow is free from the waves if the jets are not correctly expanded. The pattern of the base pressure results indicates that inside, the flow field is full of waves. The waves getting reflected after hitting the wall undergo compression and expansion, which can be noticed from Fig. 11.

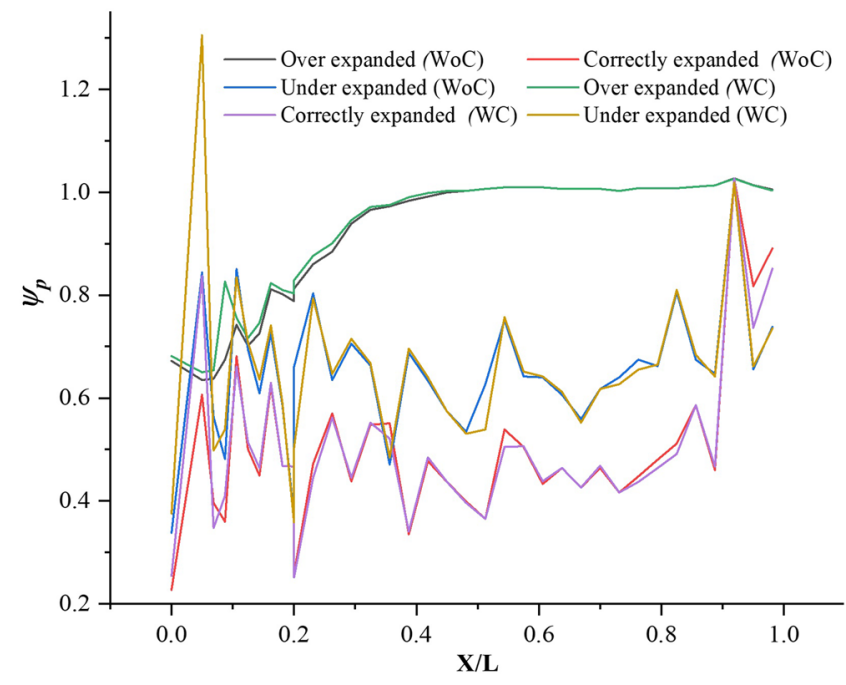

FIG. 11. Wall pressure $\left(\Psi_{p}\right)$ along the length of the duct for over-, correctly, and under-expanded flows at $M=2, \xi_{r}=5, \Lambda_{r}=2.56$, and $\phi_{r}=10$ for the cases of WC and WoC.
One of the common problems encountered in a suddenly expanded flow field is that the pressure field in the enlarged duct becomes oscillatory because of the "Ejector Pump" action. ${ }^{37}$ At the base region, the vortices are formed at the base because of the expansion of the shear layer from the nozzle and ejected to the main flow continuously. This action was referred to as the "Jet Pump-action" by Wick. ${ }^{37}$ This action renders the flow in the duct to become oscillatory. These oscillations are reflected as variations in the wall pressure distribution of the enlarged duct. Therefore, it becomes mandatory on the part of a researcher working on sudden expansion problems to monitor wall pressure distributions and the development of the flow in the enlarged duct. In other words, when we employ control to modify the base pressure level, there is a possibility that the control might augment the oscillatory nature of the flow field in the enlarged duct.

Wall pressure distribution in the enlarged duct was measured for all combinations of parameters of the present investigation to account for this undesirable effect (aggravating the oscillatory nature of the flow field). Even though voluminous data on wall pressure were collected, only some representative pressure distribution to bring out the influence of the active control on the wall pressure field is presented in Fig. 11 in order to avoid the repetition of information of similar nature. The wall pressure taps placed for the pressure recordings are shown in Fig. 12. The measured wall pressure has been made non-dimensional with the atmospheric pressure, $P_{a}$, to which the flow was discharged. The axial distance of the enlarged duct from the base location, $X$, has been non-dimensionalized with the duct length, $L$. To quantify the effect of control on wall pressure distribution $\mathrm{P}_{w} / \mathrm{P}_{a}$ for the three cases, namely (i.e., over, under, and correctly expanded jets) for with and without control, have been compared.

Figure 11 presents the static wall pressure $\left(\Psi_{p}\right)$ along the length of the duct for over-, correctly, and under-expanded flows at $M=2$ with and without control for $\xi_{r}=5, \Lambda_{r}=2.56$, and $\phi_{r}=10$. It is observed that for the wall pressure along the duct for overexpanded jets, the flow field remains identical for with and without control cases except at the entrance of the duct where the flow passes through the oblique shock, and there are mild oscillations. The level of over-expansion is low (i.e., $P_{e} / P_{a}=0.277$ ), and for $X / L>0.2$, there is a smooth recovery of the wall pressure, and it attains ambient flow conditions. When the flow expands from the nozzle into the duct, it collides with the duct wall and then gets compressed along the flow direction. This compression and expansion of flow cause rise

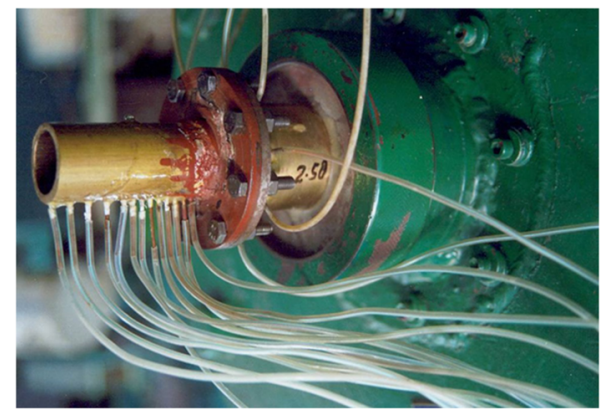

FIG. 12. Wall pressure taps at regular intervals along the duct wall. 
and fall of the pressure along the duct wall, which is observed from the wall pressure (Fig. 11). When we see the wall pressure pattern for the correctly expanded case, oscillations are observed due to the presence of the Mach waves; however, the flow field for WC and WoC remains identical. Similarly, when we analyze the wall pressure and the nature of the flow field under the influence of a favorable pressure gradient, the wall pressure results with and without control are almost identical except within the reattachment point where the flow is interacting with waves reflecting from the duct and the spike is seen where the wall pressure attains a value that is $20 \%$ more than the ambient pressure. The wall pressure for ideally expanded and under-expanded cases remains oscillatory in nature for both the cases (i.e., WC and WoC). This is one of the major advantages that the dynamic controls do not aggravate the flow field in the duct and remain identical.

\section{B. Data analysis}

Heat maps are, by definition, visual representations of data using color-coded frameworks. The main aim of heat maps is to adequately display the number of locations or events in a dataset and to aid viewers in the most relevant areas. Using the Microsoft Excel software, the heat maps are obtained for the base pressure (with and without control) and wall pressure (with control). The wall pressure is unaffected by the control using micro-jets; hence, only the readings belonging to without control are considered for data visualizing. The pressure readings are huge in number (more than $6 \mathrm{k}$ for base pressure and $0.1 \times 10^{6}$ for wall pressure); hence, data analysis is essential. Moreover, to understand the distribution of pressure, heat maps assist by sorting them based on the input variables. These huge numerical data are generally difficult to analyze; hence, the color scheme that occurs in very less space is easy to interpret. In Figs. 13-15, the heat maps of base pressure without control and with control and wall pressure are provided. The dendrograms provided for respective sections show clearly the systematic classification of data points.

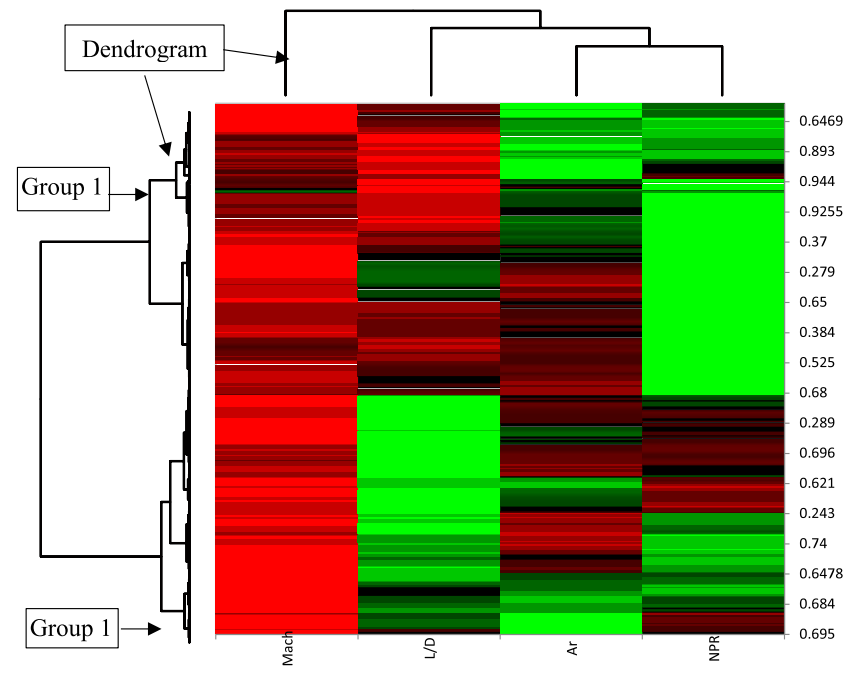

FIG. 13. Heat map of base pressure without control.

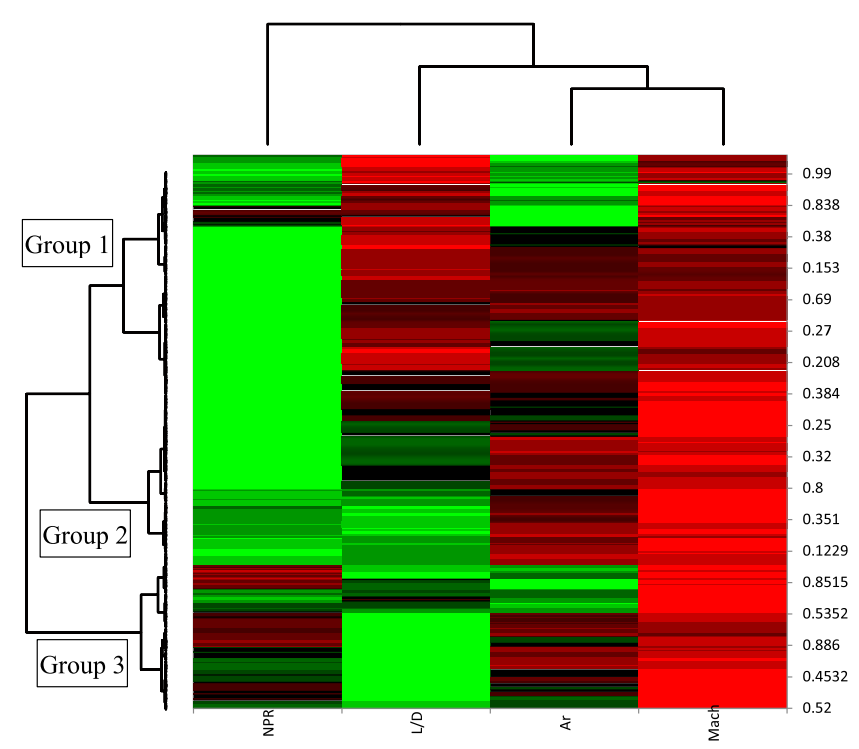

FIG. 14. Heat map of base pressure with control.

The base pressure and input variables $\left(\Lambda_{r}, M, \mathrm{NPR}\right.$, and $\left.\phi_{r}\right)$ are clustered together in Figs. 13-15. The right-side vertical axis represents the base pressure values, and the bottom horizontal axis shows the input variables. The dendrograms are for the classification of the data. The top dendrogram brings similar variables close to each other in columns, and the left dendrogram brings the same pressure values together in rows. The dendrogram clusters similar data using permutation. The heat map is a colored representation of data. The red color represents the low value, and the green color represents the high values of pressure, while the black color represents the intermediate value of the base pressure. The uppermost clustered dendrogram indicates the four branches of variables, i.e., one each

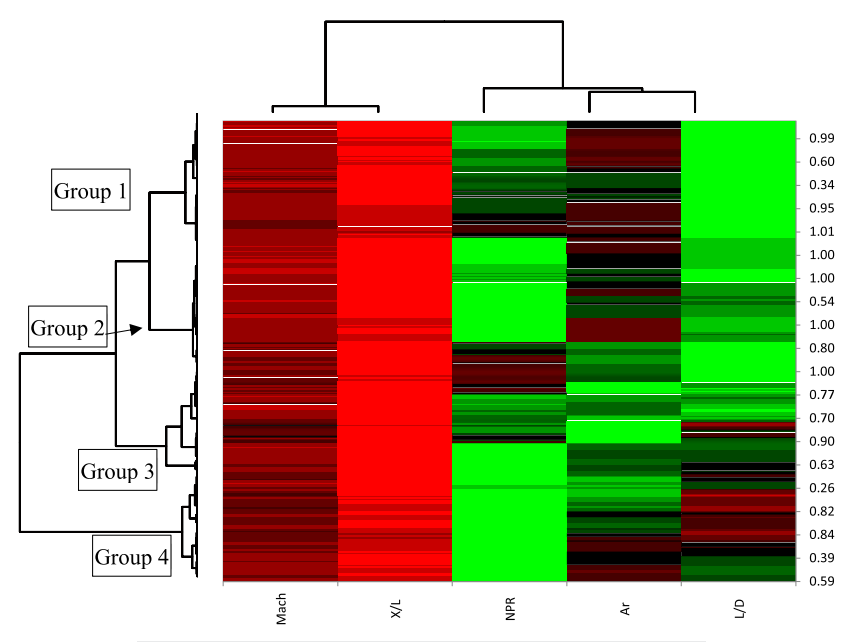

FIG. 15. Heat map of wall pressure with control (WC). 
for a single input variable chosen. For every Mach, there are $\Lambda_{r}$ values, and similarly, for every $\Lambda_{r}$, we have NPR and so on. The vertical dendrogram represents the cluster of two pressure values mainly. At this point, we have reached the most exciting part of heat maps. We will analyze whether the two pressure clusters say group 1 and group 2 (refer to Fig. 13) are associated with the input variable clusters. If we see the last column of NPR, it can be noticed that in group 1, most of the region is green in color, representing that the base pressure values are high in range. The red color is rarely spotted in the entire column showing that the base pressure belonging to lower values is less, while the black color is observed lightly. In group 2, the base pressure belongs to red and black color hence this indicates the low-pressure region.

It can be inferred that the most critical factor affecting the base pressure is NPR. Second, Mach numbers in groups 1 and 2 have only red and black colors, respectively; very rarely, the green color is spotted in group 1. This indicates that intermediate and lower values of $M$ and NPR have a prominent role in increasing the pressure. $\phi_{r}$ in group 2 indicates the higher base pressures than that of group 1. $\Lambda_{r}$ in both groups has equally given rise to the base pressure, but most of the region in both groups is related to lower and intermediate values.

In Fig. 14, the heat map of the base pressure obtained from the active control shows that the NPR and group 1 pressure cluster mostly belong to the green region, and group 2 has the green as well as black region equally. Group 3 has majorly a red zone indicating lower values of the base pressure. Group 1, having a large portion as green, means that the base pressure using control is useful in improvising it. The Mach number again here shows the same effect in intermediate and lower values as previously. $\phi_{r}$ in group 3 indicates a sufficient green region, which is clearly visible; hence, the base pressure is more in that cluster. $\Lambda_{r}$ in the sub-classification of group 1 has some region of green and most of remaining the part in the red and black zone.

In Fig. 15, the wall pressure heat map visualization is shown, obtained from the control mechanism with an extra variable, i.e., the non-dimensional duct length $X / L$. The wall pressure readings are clustered in four groups and are associated with five input variables. At this stage, it is easy to understand that the NPR again shows a prominent role in obtaining the high pressure at the duct walls. To some extent, $\Lambda_{r}$ and $\phi_{r}$ also indicated the green zones at randomly distributed rows in all groups. However, the length $X / L$ and $M$ do not show the sign of unaffected variations. This, in turn, indicates that for a specific $M$, there is a value of NPR at which the base pressure is highest. That NPR is most significant in reducing the base drag for the given nozzle and operating conditions. The nondimensional duct length indicates that the wall pressure readings are in lower zones for all the groups formed. The duct length in smaller ranges belongs to the group of wall pressure, while higher NPR and lower $M$ values belong to most of the wall pressure groups.

The histogram is a way of understanding the distribution of data when they are significant in number. As our experimental data were enormous, we decided to go for the histogram as well to know how active control affects the base pressure. For wall pressure, the control readings were preferred, and moreover, the no-control readings were also the same. The pressure is distributed in ten classes equally from 0 to 1 , as shown in Fig. 16. The density indicates how dense a class is. Base pressure histograms look similar, but a careful view suggests that the class of $0.2-0.3,0.3-0.4$, and $0.7-1.0$ the density is more for base pressure WC than WoC. This means that for some class of pressure, the density being less for flow WC indicates that the active control using micro-jets provides a boost to increase the pressure. As seen from Figs. 16(a) and 16(b), for base pressure $0-0.2$, the density is more for the case of WoC than WC. This indicates that the base pressure is more when the flow is undergoing correct expansion that is affected by active control. Hence, a reduction in the base pressure is obtained in the case of WC as the density for class $0-0.2$ has clearly reduced. The wall pressure density is shown in Fig. 16(c). The class of pressure in the range of $0.8-$ 1.0 is the highest and nearly twice the other class densities. Hence, the wall pressure is enormous for only this class, and for high pressure above 1.0, the density is low. Also, it can be concluded that the active control does not increase the wall pressure, which is a good sign.

\section{ANN regression analysis}

The regression of the base and wall pressure data is performed in different stages, as mentioned in Sec. III. A total of six backpropagation models (BPM) are proposed for the pressure WC and WoC. Additionally, a semiempirical correlation is also tried and compared to the predicted values with the BPMs. Two correlations are obtained after the rigorous use of the Microsoft Excel software for the highly sensitive and non-linear data of the base pressure. For wall pressure, the correlation is not developed as it is also a function of duct length and highly unpredictable. Equations (3) and (4) represent the developed correlations for base pressure alone,

$$
\Omega_{p}=1.6 \sin \left((0.3 M)^{\varphi_{\mathrm{r}}}+\left(\xi_{r} 0.09^{M}\right)^{\lambda}+\sin \left(\frac{0.6+0.4 \Lambda_{r} M}{\xi_{r}}\right)\right)-0.65
$$

where $\lambda$ is calculated as follows: $\lambda=\Lambda_{r}(0.3 M)^{\varphi_{r}}+\frac{M+0.4 \Lambda_{r}^{2}}{\xi_{r}}$,

$$
\Omega_{p}=(0.3 M)^{0.65+\varphi_{r}}+\frac{0.9+0.14 \Lambda_{r} M}{\xi_{r}}+\frac{0.04^{M} \xi_{r}^{2}}{\Lambda_{r}}-0.1 .
$$

Figure 17 shows the results of RMSE of all the BPMs developed, obtained after $5 \mathrm{k}$ to $10 \mathrm{k}$ iterations. It can be noted that the RMSE of each model, either WoC or WC and having a combined wall and base pressure is different. For most of the models, the RMSE value is less than 0.05 indicating less error and more suitability of developed models for prediction in the case of training and testing models. When the wall pressure and base pressure are both combined, the highly non-linear data become slightly tricky to predict the data requiring more iterations to converge.

Figure 18 shows the comparison between BPM 1, experimental results, and correlations [Eqs. (3) and (4)]. The base pressure WoC is used for training the BPM 1, and the correlations are used for prediction. There is sufficient agreement between the models and experimental readings. Out of huge training data points, only the first 100 points are used to show the pressure values from different methods clearly. For the 20 points, the zoom view is provided, which clears that the model is the most suitable one. It is seen that though both the correlations provide the same trend, the BMP 1 model provides very close results. This shows that BPM 1 is most suitable for 
(a)
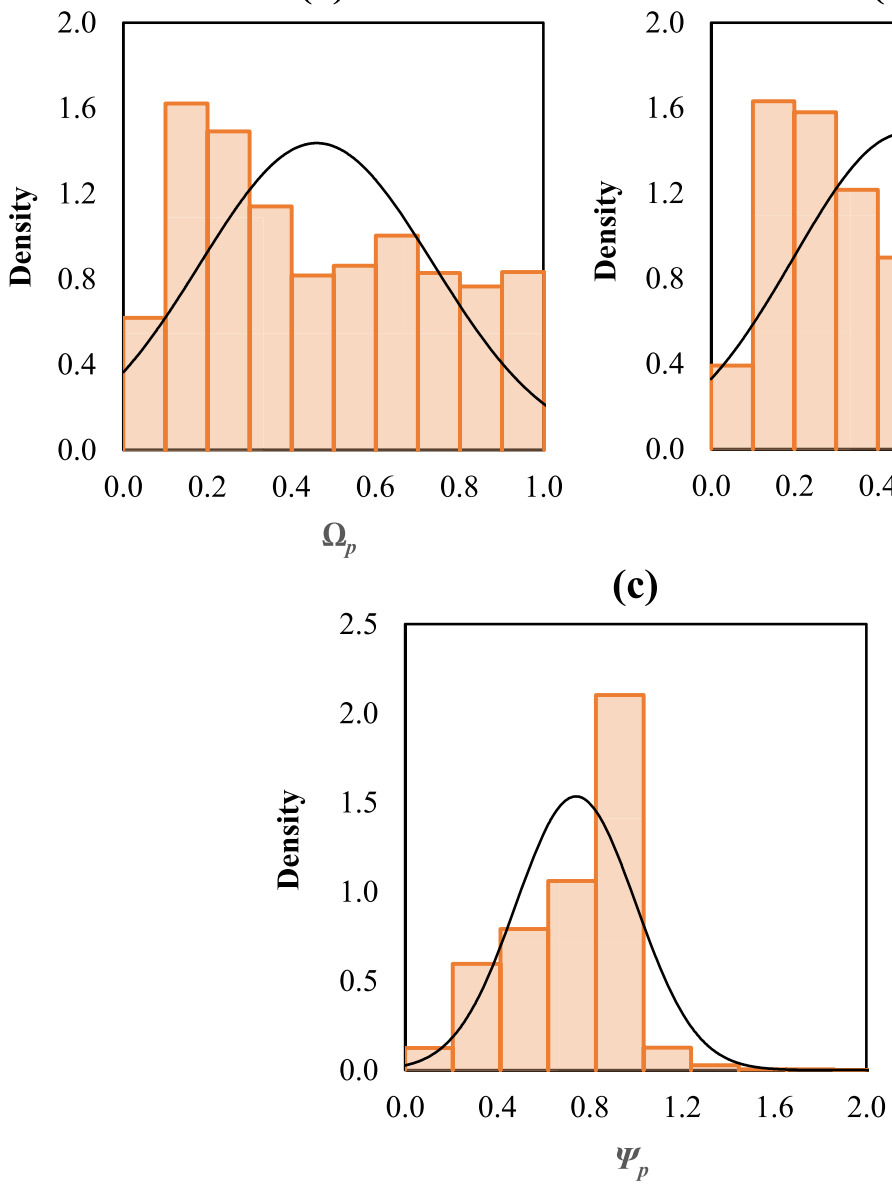

(c)

(b)

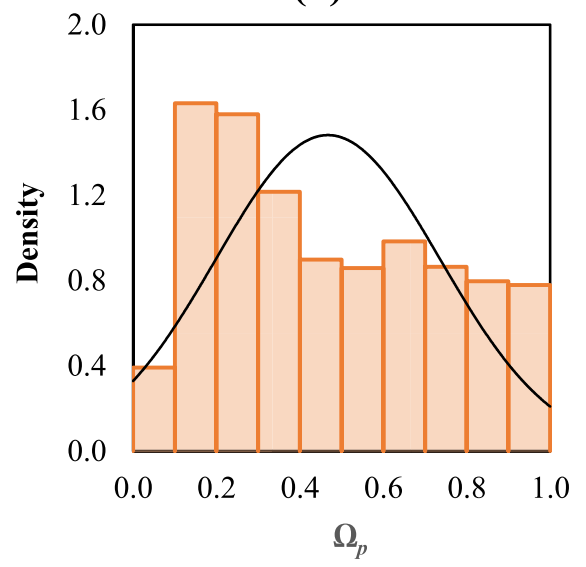

FIG. 16. Histogram plot of the base pressure for the case of (a) WoC, (b) WC, and (c) wall pressure WC.

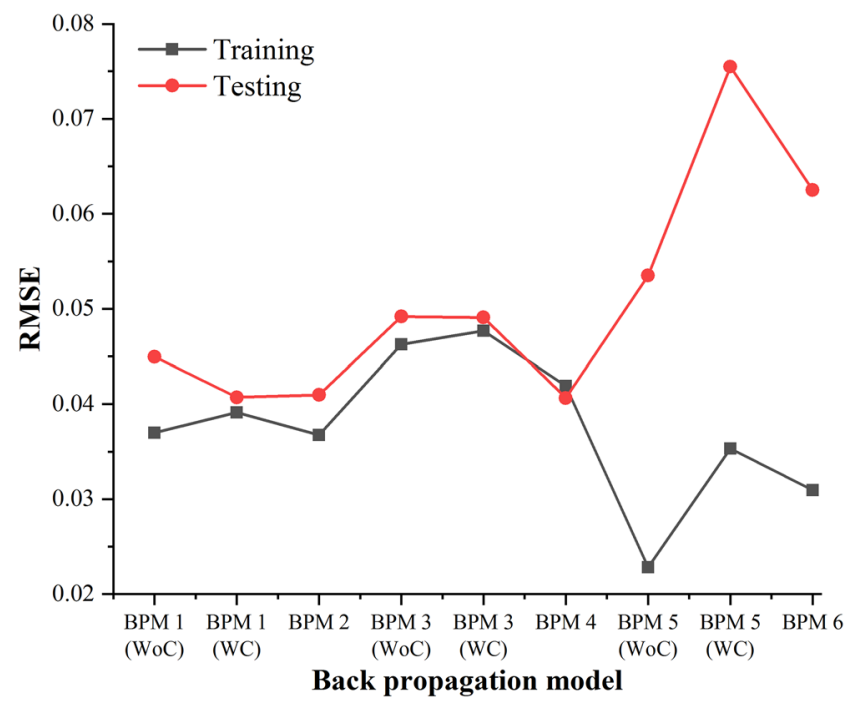

FIG. 17. RMSE obtained for each BPM for training and testing datasets. prediction than the correlations. However, these correlations can be used with a comprise in minute accuracy loss. BPM 1 is very close to the pattern of experimental data and nearly overlaps at most of the points.

In Fig. 19, the prediction of base pressure WC using the data for training and testing obtained from BMP 1 is compared with the experimental results. A good agreement between these readings is observed. The training and testing of BPM 1 are successful for pressure WC. It should be noted that for minor data points, which can be called outliers, the model does not accurately predict them, which is usually neglected. As the compressible flow data are highly nonlinear, the prediction is generally not easy. The BPMs were tried with 4-4-1, 4-4-4-1, 4-5-1, 4-5-5-1, 4-6-1, and 4-6-6-1 combinations, where the first number represents the inputs $\left(N P R, M, \Lambda_{r}\right.$, and $\left.\phi_{r}\right)$, the last number represents the output, i.e., base pressure, and the middle number represents the number of neurons in hidden layers. ANN modeling of high-speed compressible flow data is also very rare due to its non-linearity. This study to the best knowledge of authors is first to report regression modeling using ANN and to develop an analytical model for the prediction of base pressure at sonic and supersonic Mach numbers. The hidden layers finally 


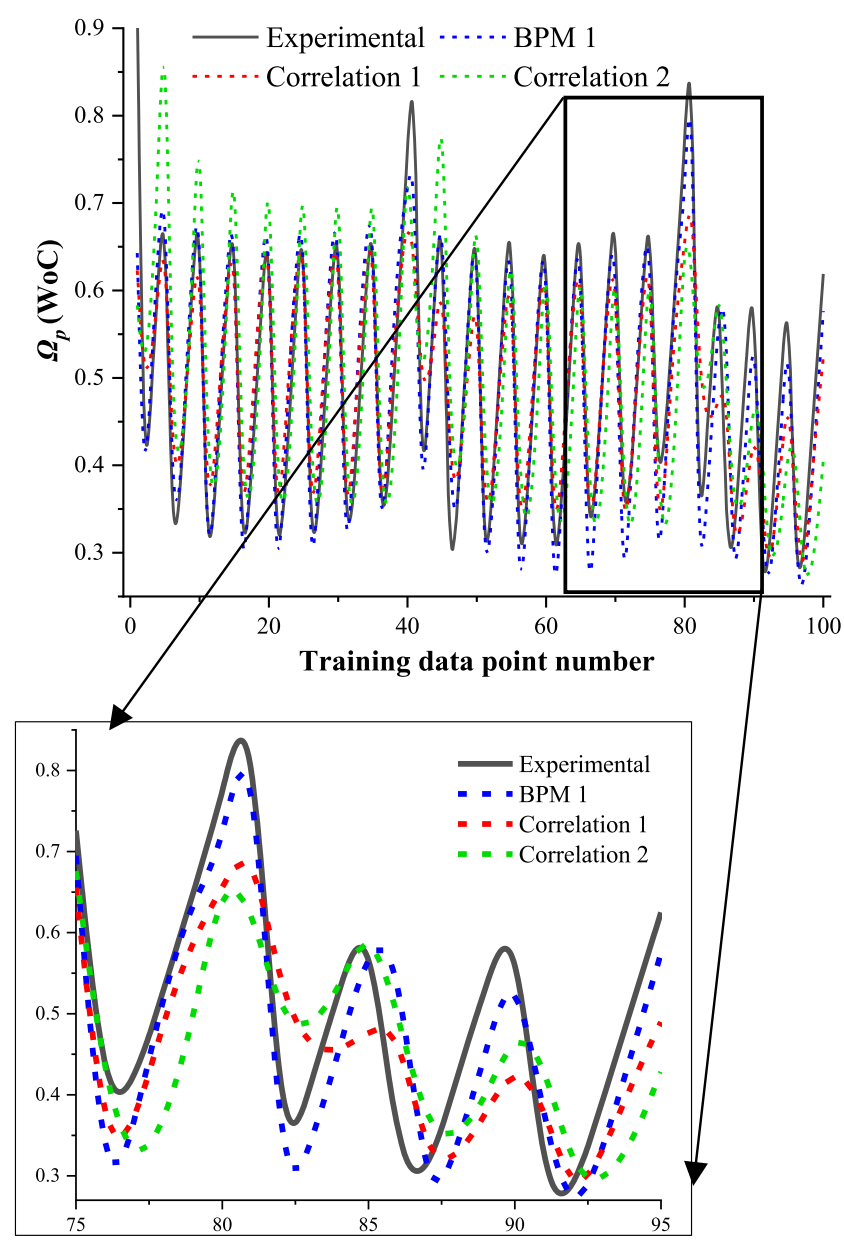

FIG. 18. Comparison of experimental, neural network model BPM 1 (without control), and correlations [Eqs. (3) and (4)].

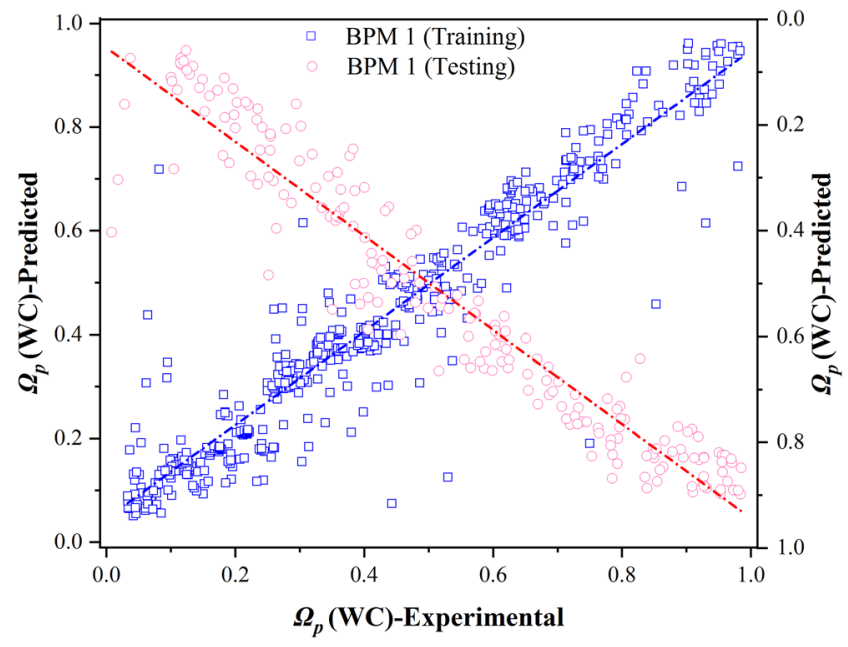

FIG. 19. Prediction of base pressure WC from BPM 1 during training/testing.

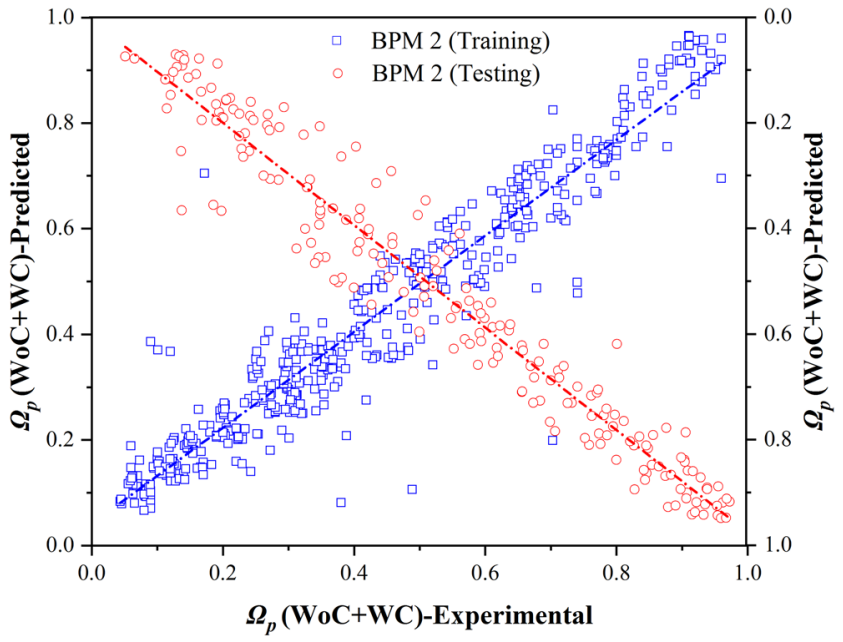

FIG. 20. Prediction of combined base pressure WC and WoC from BPM 2 during training/testing.

decided were two in number having four neurons per layer for the remaining analysis here onwards.

In Fig. 20, the training and testing of BMP 2 are depicted, which uses the base pressure from combined $\mathrm{WC}$ and $\mathrm{WoC}$ data points. The successful training of BMP 2 can be seen as predicted, and experimental data points are in-line. The testing data predicted by BPM 2 further confirm the successful prediction ability of the ANN. It can be noted that the combined WC and WoC data are higher in number, which causes a more accurate prediction of the models. In Fig. 21, the wall pressure WoC trained and tested BPM 3 data are compared with the experimental results. It is again observed that a good agreement between both the readings is obtained. The

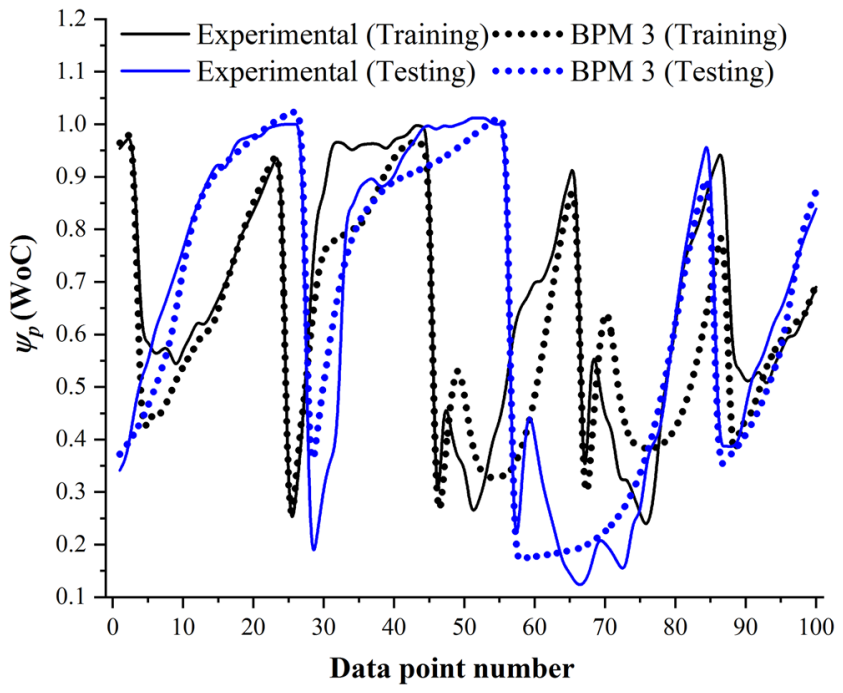

FIG. 21. Prediction of wall pressure WoC from BPM 3 during training/testing. 
highly non-linearly varying data points of wall pressure obtained WoC are successfully predicted by the model with some slight over or under prediction but acceptable. The wall pressure readings are enormous in number, as mentioned earlier, also, but very few points are shown for the sake of clarity. It is more pleasing that the testing data predicted from BPM 3 are as good as the training data prediction.

The training of BPM 3 for the wall pressure WC dataset and the corresponding prediction after thousands of iterations of backpropagation is shown in Fig. 22, which is obtained close to the experimental data. During back-propagation, the weights and bias functions of the neurons are updated in each iteration based on the selected activation function (sigmoidal function). The testing of this model after successful training is shown in the same figure [Fig. 22]. The prediction of the tested data and comparison with the experimental results reveal a good agreement between the two. The data are high in number, and the outliers as usual should be neglected. The experimental and ANN model output can be seen in-line and equally distributed along both sides.

In Fig. 23, the training and testing data points predicted by BPM 4 for wall pressure combined WC and WoC are shown. A separate model was necessary to design for the data when the pressure was combined WC and WoC. Because when the pressure is combined WC and WoC data, it will be required to separately provide input to the neural networks to indicate that control (1) or no control (0) is an input. If WC and WoC are separately modeled, then this input is not required. Hence, the analysis is made slightly complicated yet powerful with the increased data. BPM 4 also provided good training and testing prediction of wall pressures with the experimental part.

The training and testing results of BPM 5 for base pressure are shown in Fig. 24. This is a new model where the base pressure and wall pressure are the two different outputs from a single network. In this method, the wall pressure at a particular point was selected, which was decided as the reattachment point as it is of some physical significance where the flow attaches with the duct after the

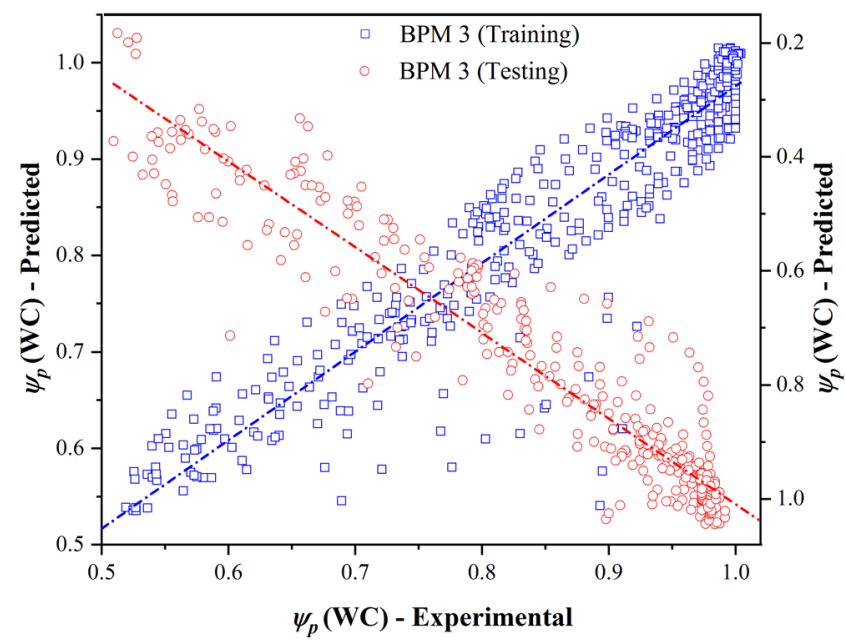

FIG. 22. Prediction of wall pressure WC from BPM 3 during training/testing

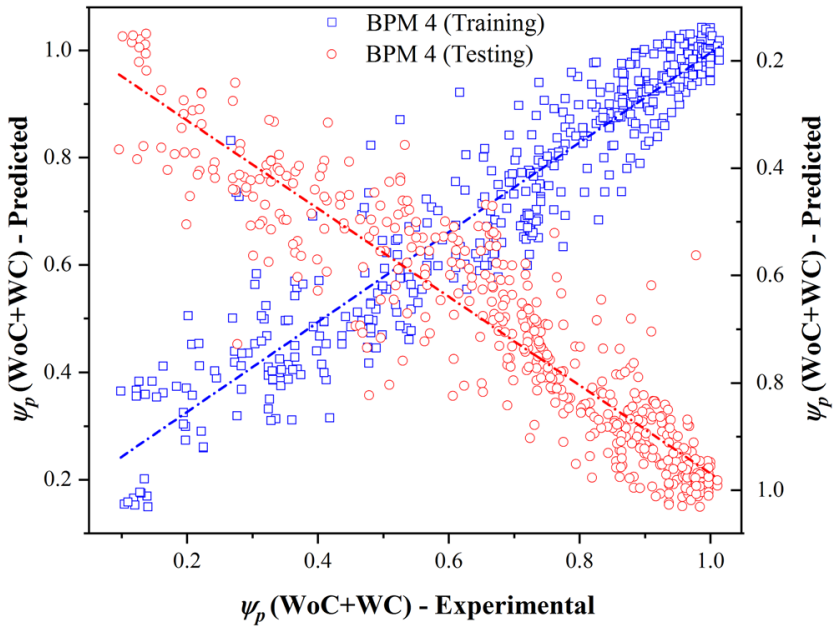

FIG. 23. Prediction of combined wall pressure WC + WoC from BPM 4 during training/testing.

nozzle exit. Hence, the wall pressure readings recorded at different points along the duct length are discarded in these models. The same method is adopted for the next model as well, where the input of $\mathrm{WC}$ and $\mathrm{WoC}$ together is considered as a separate input perceptron. The results shown in Fig. 24 show a very close match between the experimental and BPM 5. This is the most accurate model obtained so far. The increase in accuracy is only due to the increase in data points.

Figures 25-27 represent the training data prediction and testing data prediction using BPM 4 for wall pressure and base pressure when applied separately. Figure 25 is for base pressure WC showing both training and testing predictions, while Figs. 26 and 27 are

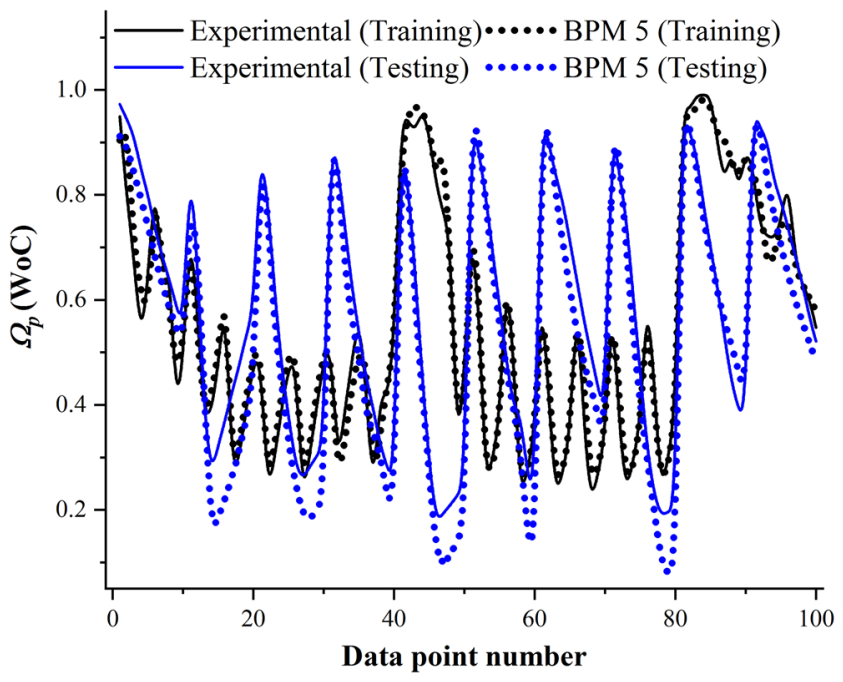

FIG. 24. Prediction of base pressure WoC from BPM 5 during training/testing. 


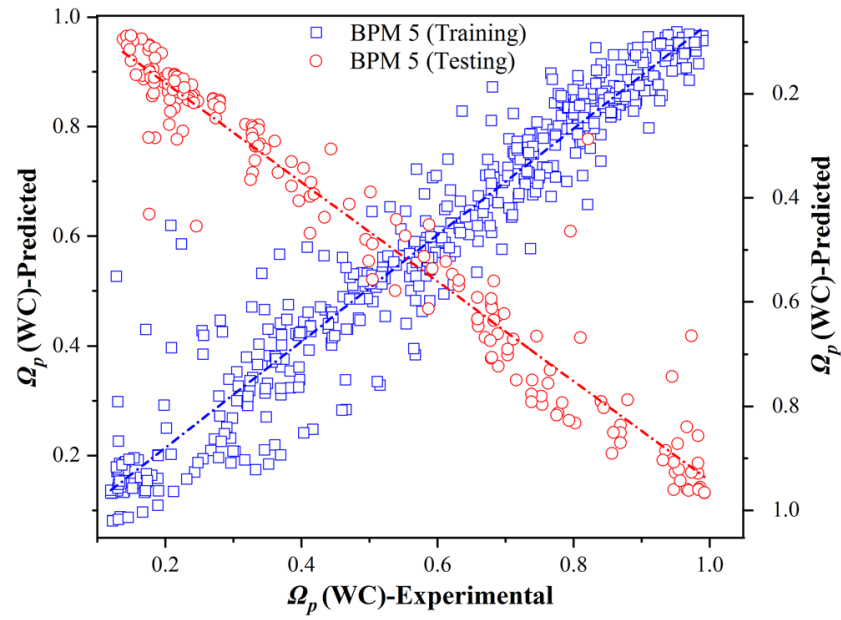

FIG. 25. Prediction of base pressure WC from BPM 5 during training/testing.

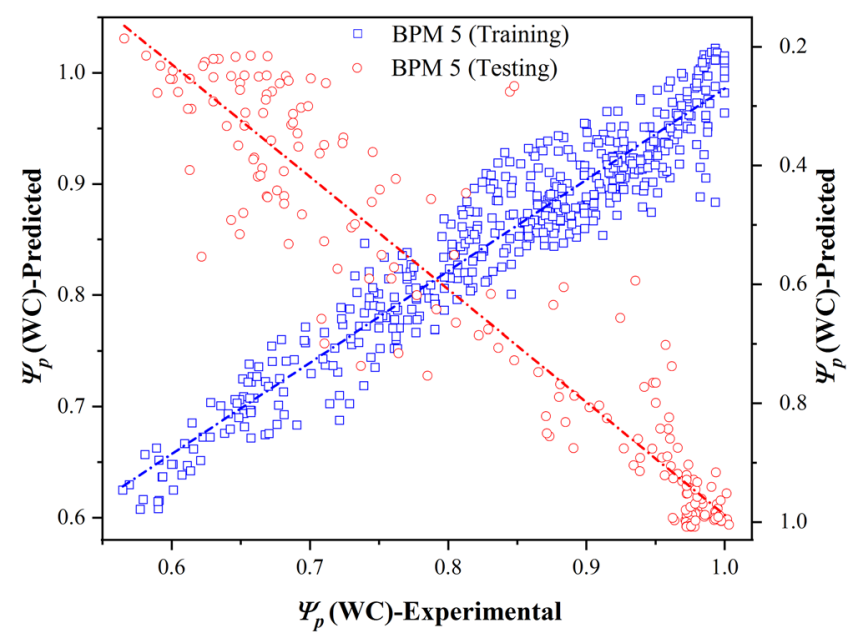

FIG. 27. Prediction of wall pressure WC from BPM 5 during training/testing. exclusively for wall pressure $\mathrm{WoC}$ and WC, respectively. As mentioned previously, BPM 5 is the most accurate model obtained until now as it is predicted very close to the experimental data. Figure 26 shows the wall pressure data WoC, which depicts a nonlinear behavior. This model BPM 5 has successfully predicted the training and testing points comfortably. Most of the data point numbers can be seen perfectly overlapping. Figure 27 also represents the same for wall pressure WC.

Figure 28 shows the training of BPM 6 when both the pressures are combined WC and WoC. It can be imagined how huge these data will be as it is the last possible combination. Due to the increased size of data, the model BPM 6 has trained even better than BPM 6 , which can be seen from Fig. 28. The trend of both the pressures from BPM 6 and experimental readings is in perfect agreement. The base pressure prediction from BPM 6 shows the ability of how a properly developed and experimented back-propagation network can easily give the values with such high accuracy. This analysis has opened a way further to explore the ability of these models in base drag, and skin friction associated with the high-speed flows.

In Fig. 29, the testing results from BPM 6 are provided for both the pressures. It can be noted that the pressure prediction from the model has very close readings above and below the trend line. This was not observed with the previous models, which showed a slightly uncomfortable result. However, with the advent of neural networks, even the highly fluctuating data were able to be modeled. The data points used to plot the predicted and desired values are fewer in number as the plot becomes complicated to understand; hence, fewer data points are shown for better clarity.

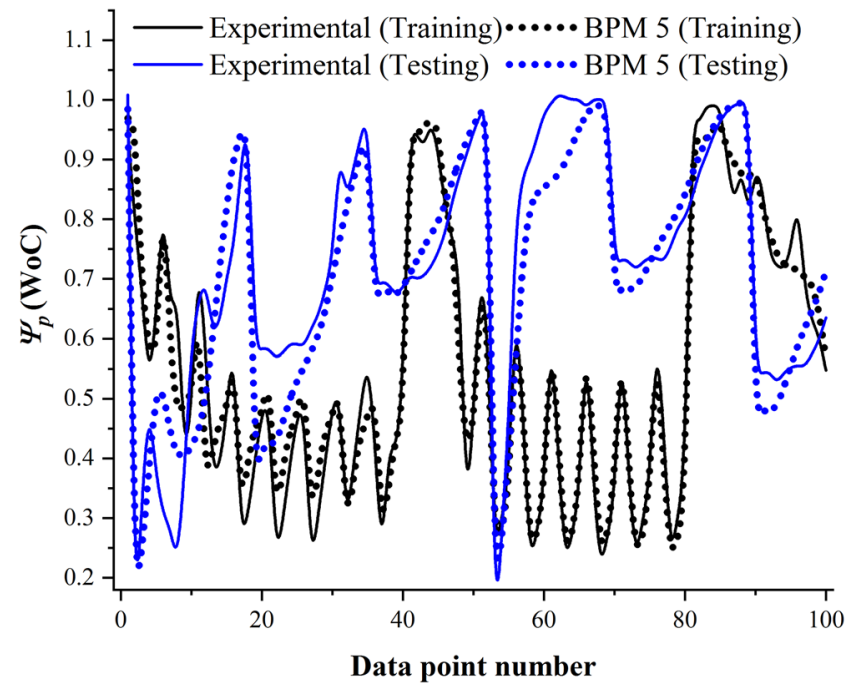

FIG. 26. Prediction of wall pressure WoC from BPM 5 during training/testing.

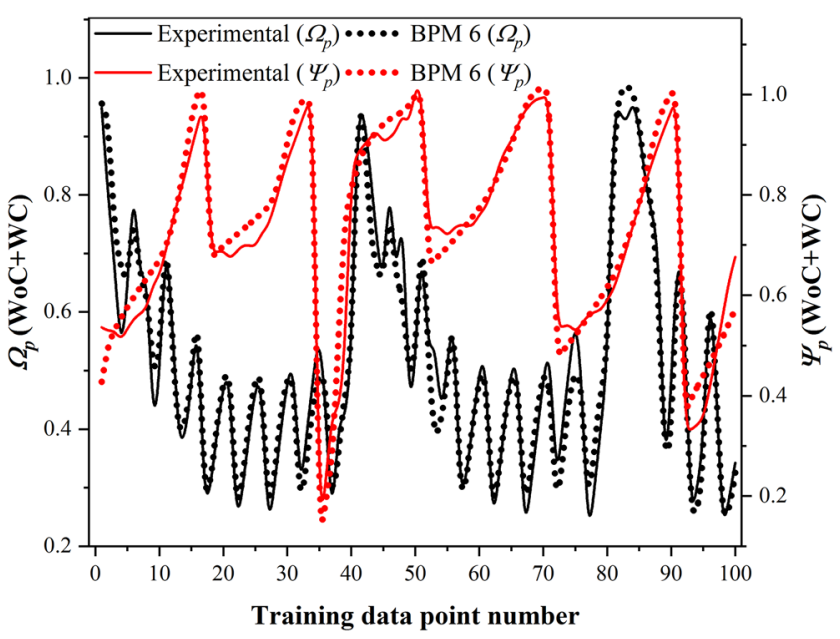

FIG. 28. Training data prediction for base pressure and wall pressure of combined WC and WoC from BPM 6. 


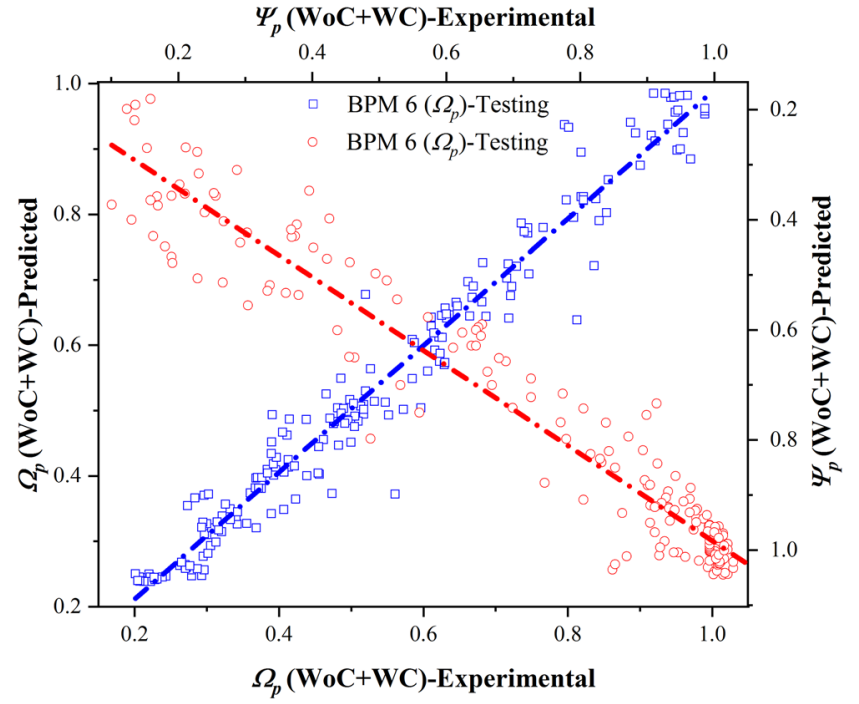

FIG. 29. Testing of BPM 6 for base pressure and wall pressure of combined WC and WoC.

\section{CONCLUSION}

The base pressure study in a rapidly expanding flow from a nozzle into an enlarged duct is investigated at sonic and supersonic Mach numbers. The other variables for the evaluation of pressure are NPR, $\phi_{r}$, and $\Lambda_{r}$. The presence of actively controlling micro-jets influenced the flow. The combination of with and without control was explored. ANN modeling of the huge data was carried out in detail by developing six back-propagation models. Data visualization was also performed. The below conclusions are derived from the inter-disciplinary analysis:

- The base pressure increases with the increase of Mach number and is also found to be severely affected by NPR. The amount of growth in the base pressure is purely a factor of Mach and NPR. For NPR below the correct expansion, the base pressure reduces from high to lower due to the formation of shock waves.

- When the NPR is above the perfect expansion, the base pressure increases for low to high. $\phi_{r}$ and $\Lambda_{r}$ have a marginal effect on the base pressure. For lower $\Lambda_{r}$, the pressure reduces, and for high $\Lambda_{r}$, the pressure increases magnificently due to an increase in the size of the primary vortex at the base.

- Based on NPR for over-expansion, the pressure is higher; for perfect expansion, it is low; and for under-expansion, the pressure is again high. The rise and fall of pressure are highly non-linear.

- With the use of active micro-jets, the base pressure reduces in the zone of over-expansion and increases in the zone of under-expansion. The wall pressure remains unaffected by the employment of micro-jets.

- The data analysis using a heat map revealed some essential facts. NPR is the most influential parameter to affect the base pressure, while the lower range and middle range of Mach numbers (used in this experiment) provide the maximum base pressure. The heat map also revealed that the area ratio has minor effects, while the $\phi_{r}$ ratio also significantly increases the base pressure.

- The highly non-linear data obtained pertinent to the base and wall pressure is modeled by the back-propagation neural networks. Six BPMs are developed, and each model with acceptable prediction gives the pressure data. However, BMP 5 and BPM 6 are found to be most suitable for both the pressures, which more accurately predict and are found to be in excellent agreement with the experimental data.

\section{DATA AVAILABILITY}

The data that support the findings of this study are available from the corresponding author upon reasonable request.

\section{REFERENCES}

${ }^{1}$ S. A. Khan and E. Rathakrishnan, "Active control of suddenly expanded flows from overexpanded nozzles," Int. J. Turbo Jet Engines 19, 119-126 (2002).

${ }^{2}$ S. A. Khan and E. Rathakrishnan, "Control of suddenly expanded flows with micro-jets," Int. J. Turbo Jet Engines 20, 63-81 (2003).

${ }^{3}$ S. A. Khan and E. Rathakrishnan, "Control of suddenly expanded flows from correctly expanded nozzles,” Int. J. Turbo Jet Engines 21, 255-278 (2004).

${ }^{4}$ S. A. Khan and E. Rathakrishnan, "Active control of suddenly expanded flows from underexpanded nozzles," Int. J. Turbo Jet Engines 21, 233-253 (2004).

${ }^{5}$ S. A. Khan and E. Rathakrishnan, "Active control of suddenly expanded flows from underexpanded nozzles-Part II,” Int. J. Turbo Jet Engines 22, 163-184 (2005).

${ }^{6}$ S. A. Khan and E. Rathakrishnan, "Nozzle expansion level effect on suddenly expanded flow,” Int. J. Turbo Jet Engines 23, 233-258 (2006).

${ }^{7}$ S. A. Khan and E. Rathakrishnan, "Active control of base pressure in supersonic regime,” J. Aerosp. Eng. 87, 1-8 (2006).

${ }^{8}$ S. A. Khan and E. Rathakrishnan, "Control of suddenly expanded flow," Aircr. Eng. Aerosp. Technol. 78(4), 293-309 (2006).

${ }^{9}$ Y. Zhu, W. Zhu, D. Gu, S. Chen, C. Lee, and E. S. Oran, "Acoustic-waveinduced cooling in onset of hypersonic turbulence,” Phys. Fluids 32(1), 061702 (2020).

${ }^{10}$ A. Mittal and S. S. Girimaji, "Nonlinear evolution of perturbations in high Mach number wall-bounded flow: Pressure-dilatation effects," Phys. Fluids 32(3), 036101 (2020).

${ }^{11} \mathrm{C}$. Li, Y. Zhang, and C. Lee, "Influence of glow discharge on evolution of disturbance in a hypersonic boundary layer: The effect of first mode," Phys. Fluids 32(5), 051701 (2020).

${ }^{12}$ W. Zhu, M. Shi, Y. Zhu, and C. Lee, "Experimental study of hypersonic boundary layer transition on a permeable wall of a flared cone," Phys. Fluids 32(1), 011701 (2020).

${ }^{13} \mathrm{H}$. Korst, "A theory of base pressure in transonic and supersonic flow," J. Appl. Mech. 23(4), 593-600 (1956).

${ }^{14}$ J. S. Anderson and T. J. Williams, "Base pressure and noise produced by the abrupt expansion of air in a cylindrical duct," J. Mech. Eng. Sci. 10(3), 262-268 (1968).

${ }^{15}$ M. A. Ullah, M. Bashir, A. Janvekar, and S. A. Khan, "Active control of wall pressure flow field at low supersonic Mach numbers," IOSR J. Mech. Civ. Eng. 16, 90-98 (2016)

${ }^{16}$ G. M. Fharukh Ahmed, M. A. A. Baig, M. Bashir, and S. A. Khan, "Control of ideally expanded and under expanded nozzle flows with micro jets," IOSR J. Mech. Civ. Eng. 16, 83-89 (2016). 
${ }^{17}$ S. A. Khan, M. Bashir, G. M. Fharukh Ahmed, and M. A. Ullah, "An investigation of base flow control by wall pressure analysis in a suddenly expansion nozzle," J. Sci. Res. Dev. 3(5), 1-6 (2016).

${ }^{18}$ S. A. Khan, M. A. Fatepurwala, K. N. Pathan, P. Dabeer, and M. A. Baig, "CFD analysis of human powered submarine to minimize drag ratio (L/D)," Int. J. Mech. Prod. Eng. Res. Dev. 8(3), 1057-1066 (2018).

${ }^{19}$ S. A. Khan, M. Asadullah, G. M. Fharukh Ahmed, A. Jalaluddeen, and A. Ahmed, and M. Baig, "Flow control with aerospike behind bluff body," Int. J. Mech. Prod. Eng. Res. Dev. 8(3), 1001-1008 (2018).

${ }^{20}$ A. Saleel, M. A. Baig, and S. A. Khan, "Experimental investigation of the base flow and base pressure of sudden expansion nozzle," IOP Conf. Ser.: Mater. Sci. Eng. 370, 012052 (2018).

${ }^{21}$ M. Ahmed, A. Baig, S. A. Khan, A. S. C., and E. Rathakrishnan, "Control of base flows with micro jet for area ratio of 6.25," ARPN J. Eng. Appl. Sci. 7(8), 992-1002 (2012).

${ }^{22}$ K. Vijayaraja, C. Senthilkumar, S. Elangovan, and E. Rathakrishnan, "Base pressure control with annular ribs," Int. J. Turbo Jet Engines 31(2), 111-118 (2014).

${ }^{23}$ E. Rathakrishnan, "Effect of ribs on suddenly expanded flows," AIAA J. 39(7), 1402-1404 (2001).

${ }^{24}$ C. Raibaudo, P. Zhong, B. R. Noack, and R. J. Martinuzzi, "Machine learning strategies applied to the control of a fluidic pinball," Phys. Fluids 32(1), 015108 (2020).

${ }^{25}$ Z. Ansari, A. R. Faizabadi, and A. Afzal, "Fuzzy c-least medians clustering for discovery of web access patterns from web user sessions data," Intell. Data Anal. 21(3), 553-575 (2017).

${ }^{26}$ A. Afzal, Z. Ansari, A. R. Faizabadi, and M. K. Ramis, "Parallelization strategies for computational fluid dynamics software: State of the art review," Arch. Comput. Methods Eng. 24(2), 337-363 (2017).

${ }^{27}$ R. Jagannath, N. G. Naresh, and K. M. Pandey, "Studies on pressure loss in sudden expansion in flow through nozzles : A fuzzy logic approach," ARPN J. Eng. Appl. Sci. 2(2), 50-61 (2007).
${ }^{28} \mathrm{~K}$. M. Pandey and S. Kumar, "Flow through nozzle in sudden expansion in cylindrical ducts with area ratio 2.89 at Mach 2.4: A fuzzy logic approach," Int. J. Innovation, Manage. Technol. 1(3), 305-311 (2010).

${ }^{29}$ K. M. Pandey and A. Kumar, "Studies on base pressure in suddenly expanded circular ducts: A fuzzy logic approach," Int. J. Eng. Technol. 2(4), 379-386 (2010).

${ }^{30}$ K. M. Pandey, J. Rajshekharan, and S. Roga, "Wall static pressure variation in sudden expansion in flow through De Laval nozzles at Mach 1.74 and 2.23: A fuzzy logic approach," in International Conference on Machine Learning and Computing, January 2010 (IJSCE, 2010), pp. 243-247.

${ }^{31}$ K. M. Pandey, S. Kumar, and J. P. Kalita, "Wall static pressure variation in sudden expansion in cylindrical ducts with supersonic flow: A fuzzy logic approach," Int. J. Soft Comput. Eng. 2(1), 237-242 (2012).

${ }^{32}$ S. R. Mounce, J. B. Boxall, and J. Machell, "Development and verification of an online artificial intelligence system for detection of bursts and other abnormal flows," J. Water Resour. Plann. Manage. 136(3), 309-318 (2010).

${ }^{33}$ N. R. Secco and B. S. de Mattos, "Artificial neural networks to predict aerodynamic coefficients of transport airplanes," Aircr. Eng. Aerosp. Technol. 89 (2), 211-230 (2017).

${ }^{34}$ A. Nejat, S. A. V. Esfehani, and A. Doostmohammadi, “Airfoil flow separation and stall prediction using a neural network approach," in ASME 2010 10th Biennial Conference on Engineering Systems Design and Analysis (ASME, 2017), pp. $1-7$.

${ }^{35}$ J. Quadros, S. Khan, and A. J. Antony, "Predictive modeling of suddenly expanded flow process in the supersonic Mach number regime using response surface methodology," Int. J. Recent Res. Asp. 49(1), 149-160 (2018).

${ }^{36} \mathrm{~J}$. Quadros, S. Khan, and A. J. Antony, "Modelling of suddenly expanded flow process in supersonic Mach regime using design of experiments and response surface methodology," J. Comput. Appl. Mech. 49(1), 149-160 (2018).

${ }^{37}$ R. S. Wick, "The effect of boundary layer on sonic flow through an abrupt crosssectional area change," J. Aeronaut. Sci. 20, 675-682 (1953). 